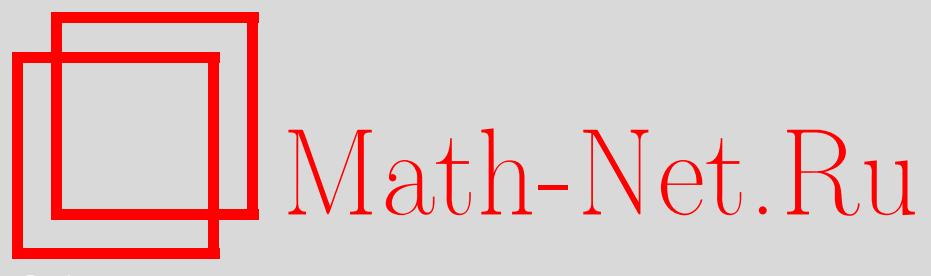

М. Г. Плотников, Некоторые свойства многомерных обобщенных интегралов и теоремы типа Дю БуаРеймона для двойных рядов Хаара, Матем. сб., 2007, том 198, номер 7, 63-90

DOI: https://doi.org/10.4213/sm1506

Использование Общероссийского математического портала Math-Net.Ru подразумевает, что вы прочитали и согласны с пользовательским соглашением http://www . mathnet.ru/rus/agreement

Параметры загрузки:

IP : 52.90 .164 .192

26 апреля 2023 г., 12:01:11 


\section{М. Г. Плотников \\ Некоторые свойства многомерных обобщенных интегралов и теоремы типа Дю Буа-Реймона для двойных рядов Хаара}

Рассматривается ряд свойств многомерных обобщенных интегралов. В двумерном случае доказывается непротиворечивость регулярного интеграла Перрона и одного обобщенного интеграла, решающего проблему восстановления коэффициентов двойных рядов Хаара из некоторого класса. Как следствие, получен ряд обобщений известной теоремы Скворцова. В частности, получен следующий результат. Если для некоторого $\rho \in(0,1 / 2]$ двойной ряд Хаара всюду на единичном квадрате $\rho$-регулярно сходится к конечной функции, интегрируемой по Перрону в $\rho$-регулярном смысле, то данный ряд есть ряд Фурье-Перрона своей суммы.

Библиография: 20 названий.

\section{§1. Введение}

Классическая теорема Дю Буа-Реймона (см. [1], [2; гл. 1]) утверждает, что тригонометрический ряд, всюду сходящийся к интегрируемой по Риману функции, является рядом Фурье этой функции. Этот результат обобщался на случай других интегралов и переносился на ряды по другим ортонормированным системам.

Здесь мы доказываем теоремы такого типа для двойных рядов Хаара

$$
\sum_{n=1}^{\infty} \sum_{m=1}^{\infty} a_{n, m} \chi_{n, m}(x, y) .
$$

В этом направлении известны результаты В. А. Скворцова (см., например, [3; теорема 2], [4]; см. также [5; теорема 2]), из которых, в частности, следует, что если ряд (1) всюду на $[0,1]^{2}$ сходится по прямоугольникам к конечной функции $s(x)$, интегрируемой в смысле нерегулярного интеграла Перрона (определение см. ниже), то данный ряд является рядом Фурье-Перрона функции $s(x)$.

Мы рассмотрим так называемую $\rho$-регулярную сходимость, которая обобщает сходимость по прямоугольникам и иногда называется ограниченной сходимостъю по прямоуголъникам. Кроме того, рассмотрим $\rho$-регулярный интеграл

Работа выполнена при поддержке Российского фонда фундаментальных исследований (грант №05-01-00206), Программы поддержки ведущих научных школ РФ (грант № НШ4681.2006.1) и Программы поддержки молодых ученых (грант № MK-1214.2005.1).

(C) М. Г. Плотников, 2007 
Перрона, который шире нерегулярного интеграла Перрона. Основным результатом работы является обобщение приведенной выше теоремы В. А. Скворцова на случай $\rho$-регулярной сходимости и $\rho$-регулярного интеграла Перрона. Этот результат будет получен как следствие более общего факта, имеющего отношение к теории интеграла. А именно, в двумерном случае будет доказана непротиворечивость $\rho$-регулярного интеграла Перрона и $\left(P_{d}^{\rho, *}\right)$-интеграла, построенного в [6] для восстановления коэффициентов рядов (1) из некоторого класса.

\section{§ 2. Определения из теории рядов Хаара}

Под мерой мы понимаем меру Лебега в $\mathbb{R}^{2}$. Расстояние между точками понимается в евклидовой метрике. Понятия открытости и замкнутости множества рассматриваются относительно топологии единичного квадрата $[0,1]^{2}$; так, $A^{\circ}$ обозначает внутренность множества $A$ в этой топологии. Под измеримостью множеств понимается принадлежность к $\sigma$-алгебре множеств, порожденной открытыми интервалами из $[0,1]^{2}$.

Мы используем стандартное определение одномерных функций Xaара $\chi_{n}(x)$, при котором система Хаара полна в $C[0,1]$ (см. [7], [8]), т.е. $\chi_{1}(x) \equiv 1$ на $[0,1]$, а при $n=2^{k}+i, k \geqslant 0,1 \leqslant i \leqslant 2^{k}$

$$
\chi_{n}(x)= \begin{cases}2^{k / 2} & \text { при } x \in\left(\frac{2 i-2}{2^{k+1}}, \frac{2 i-1}{2^{k+1}}\right), \\ -2^{k / 2} & \text { при } x \in\left(\frac{2 i-1}{2^{k+1}}, \frac{2 i}{2^{k+1}}\right), \\ 0 & \text { вне }\left[\frac{2 i-2}{2^{k+1}}, \frac{2 i}{2^{k+1}}\right] .\end{cases}
$$

В точке 0 (точке 1 ) значение функции $\chi_{n}(x)$ равно ее пределу справа (слева), а в остальных точках отрезка $[0,1]$ - среднему арифметическому правого и левого пределов.

Далее $Q_{d}$ будет обозначать множество двоично-рациональных точек отрезка $[0,1]$, т.е. точек вида $p / 2^{n}$, где $n \geqslant 0, p=\overline{1,2^{n}}$. Точки множества $I_{d} \stackrel{\text { def }}{=}[0,1] \backslash Q_{d}$ называются двоично-иррачиональными.

Двумерным двоичным интервалом назовем замкнутый сегмент вида

$$
\left[\frac{p_{1}}{2^{k_{1}}}, \frac{p_{1}+1}{2^{k_{1}}}\right] \times\left[\frac{p_{2}}{2^{k_{2}}}, \frac{p_{2}+1}{2^{k_{2}}}\right],
$$

где $k_{1}, k_{2}=0,1, \ldots, p_{i}=0, \ldots, 2^{k_{i}}-1$. Пару $\left(k_{1}, k_{2}\right)$ будем называть рангом двоичного интервала (2). Если $k_{1}=k_{2}=n$, то такой двоичный интервал назовем двоичным квадратом, а его рангом будем считать число $n$. Данные понятия естественным образом обобщаются на $M$-мерный случай.

Последовательность одномерных двоичных интервалов $\left\{\Delta_{k}\right\}_{k=0}^{\infty}$ назовем основной для точки $a \in[0,1]$, если $a \in \Delta_{k}$ для всех $k=0,1, \ldots$, причем ранг $\Delta_{k}$ 
равен $k$ и $\Delta_{k+1} \subset \Delta_{k}$. Если же $\mathbf{z}=\left(z_{1}, z_{2}\right) \in[0,1]^{2}$, то двойную последовательность двумерных двоичных интервалов $\left\{\Delta_{k_{1}, k_{2}}\right\}$ назовем (z)-последовательностъю, если $\Delta_{k_{1}, k_{2}}=\Delta_{k_{1}} \times \Delta_{k_{2}},\left\{\Delta_{k_{i}}\right\}$ - последовательность, основная для точки $z_{i}$. Для каждой точки $\mathbf{z} \in[0,1]^{2}$ существует ровно $2^{s}(\mathbf{z})$-последовательностей, где $s$ - число $Q_{d}$-координат точки $\mathbf{z}$, отличных от нуля и единицы. Из $(\mathbf{z})$-последовательности выделим подпоследовательность $\left\{\Delta_{n} \stackrel{\text { def }}{=} \Delta_{n, n}\right\}$, состоящую из двоичных квадратов, которую будем называть $(\mathbf{z}, \square)$-последовательностью.

Двумерная функиия Хаара $\chi_{n, m}\left(z_{1}, z_{2}\right)$ равна $\chi_{n}\left(z_{1}\right) \chi_{m}\left(z_{2}\right)$. Прямоугольная частичная сумма ряда $(1)$ с номером $\mathbf{m}=(N, M)$ в точке $(x, y)$ есть выражение

$$
S_{\mathbf{m}}(\mathbf{z})=S_{N, M}\left(z_{1}, z_{2}\right) \stackrel{\text { def }}{=} \sum_{n=1}^{N} \sum_{m=1}^{M} a_{n, m} \chi_{n, m}\left(z_{1}, z_{2}\right) .
$$

Назовем параметром регулярности интервала $\Delta$ число $\operatorname{reg}(\Delta)$, равное отношению длин минимальной и максимальной сторон интервала, и, аналогично, параметром регулярности вектора $\mathbf{m}$ - число $\operatorname{reg}(\mathbf{m})$, равное отношению минимальной и максимальной его компонент. Интервал $\Delta \mathrm{c} \operatorname{reg}(\Delta) \geqslant \rho$ будем называть $\rho$-регулярным. Ряд (1) сходится $\rho$-регулярно $(\rho \in(0,1])$ к сумме $S(\mathbf{z})$ в точке $\mathbf{z}$, если $S_{\mathbf{m}}(\mathbf{z})$ стремится к $S(\mathbf{z})$ при $\min \{N, M\} \rightarrow \infty$ и $\operatorname{reg}(\mathbf{m}) \geqslant \rho$. Отбросив последнее условие, получим сходимость по прямоугольникам. При любом $\rho \in(0,1] \rho$-регулярная сходимость является более общей, чем сходимость по прямоугольникам.

\section{§ 3. Определения и некоторые факты из теории интеграла}

Пусть $\mathscr{B}$ - семейство всех замкнутых сегментов, лежащих в $[0,1]^{M}$. Если $\rho \in$ $(0,1]$, то $\mathscr{B} \rho$ будет обозначать семейство всех замкнутых сегментов $\Delta \subset[0,1]^{M}$ $\mathrm{c} \operatorname{reg}(\Delta) \geqslant \rho$, а $\mathscr{B}_{\rho, d}-$ семейство двоичных интервалов $\Delta \subset[0,1]^{M} \operatorname{creg}(\Delta) \geqslant \rho$. Очевидно, $\mathscr{B}_{\rho, d} \subset \mathscr{B}_{\rho} \subset \mathscr{B}$.

Два множества называются неперекрывающимися, если их внутренности не пересекаются. Функция множества $\Phi(\Delta)$, определенная для всех $\Delta \in \mathscr{B}$, называется супераддитивной (обозначение $\Phi \in \bar{A}_{\mathscr{B}}$ ), если для любых $\Delta, \Delta_{1}, \ldots$, $\Delta_{p} \in \mathscr{B}$ таких, что $\Delta=\bigcup_{j=1}^{p} \Delta_{j}$ и множества $\Delta_{j}$ попарно не перекрываются, выполнено условие $\sum_{i=1}^{p} \Phi\left(\Delta_{i}\right) \leqslant \Phi(\Delta)$. Функция $\Phi(\Delta)$ называется субаддитивной (обозначение $\Phi \in \underline{A}_{\mathscr{B}}$ ), если функция $-\Phi(\Delta)$ является супераддитивной. Функция $\Phi(\Delta)$ называется аддитивной (обозначение $\Phi \in A_{\mathscr{B}}$ ), если $\Phi \in \bar{A}_{\mathscr{B}} \cap \underline{A}_{\mathscr{B}}$. Эти понятия естественным образом переносятся и на случай функций, определенных лишь на подсемействах семейства $\mathscr{B}$.

Семейство $\mathscr{B}$ обладает тем свойством, что для любой точки $\mathbf{z} \in[0,1]^{M}$ и любого $\varepsilon>0$ найдется множество $\Delta \in \mathscr{B}$ диаметра меньшего, чем $\varepsilon$, содержащее точку z. Производная функиии множества $\Phi(\Delta)$ в точке $\mathbf{z}$ относительно семейства $\mathscr{B}$ (обозначение $\left.D_{\mathscr{B}} \Phi(\mathbf{z})\right)$ определяется равенством

$$
D_{\mathscr{B}} \Phi(\mathbf{z})=\lim _{\mathbf{z} \in \Delta, \Delta \in \mathscr{B}} \frac{\Phi(\Delta)}{|\Delta|} .
$$


Естественным образом определяются верхняя производная $\bar{D}_{\mathscr{B}} \Phi(\mathbf{z})$ и нижняя производная $\underline{D}_{\mathscr{B}} \Phi(\mathbf{z})$ функции $\Phi(\Delta)$ в точке $\mathbf{z}$ относительно семейства $\mathscr{B}$. Аналогично определяется производная функции $\Phi(\Delta)$ в точке $\mathbf{z}$ относительно любого из подсемейств $\mathscr{B} \rho$ или $\mathscr{B} \rho, d$.

ОПРЕдЕЛЕниЕ 1. Пусть на $[0,1]^{M}$ задана почти всюду конечная функция $f(\mathbf{z})$. Функция $f(\mathbf{z})$ называется интегрируемой по Перрону в нерегулярном смысле, если для всякого $\varepsilon>0$ найдутся функции интервала $F_{1} \in \bar{A}_{\mathscr{B}}$ (мажоранта) и $F_{2} \in \underline{A}_{\mathscr{B}}($ миноранта) такие, что:

1) всюду на $[0,1]^{M}$ выполнены соотношения

$$
\underline{D}_{\mathscr{B}} F_{1}(\mathbf{z}) \geqslant f(\mathbf{z}) \geqslant \bar{D}_{\mathscr{B}} F_{2}(\mathbf{z}), \quad \underline{D}_{\mathscr{B}} F_{1}(\mathbf{z})>-\infty, \quad \bar{D}_{\mathscr{B}} F_{2}(\mathbf{z})<+\infty ;
$$

2) $F_{1}\left([0,1]^{M}\right)-F_{2}\left([0,1]^{M}\right)<\varepsilon$.

Нерегулярный интеграл Перрона функции $f(\mathbf{z})$ по любому сегменту $\Delta \in \mathscr{B}$ определяется формулой

$$
(P) \int_{\Delta} f(\mathbf{z}) d \mathbf{z}=\inf _{F_{1}} F_{1}(\Delta)=\sup _{F_{2}} F_{2}(\Delta) .
$$

Пусть $P$ обозначает класс функций, интегрируемых по Перрону в нерегулярном смысле. Заменив в определении 1 семейство $\mathscr{B}$ на семейство $\mathscr{B}_{\rho}\left(\mathscr{B}_{\rho, d}\right)$, получим понятие $\rho$-регулярного интеграла Перрона (двоичного о-регулярного интеграла Перрона). Пусть $P_{\rho}\left(P_{\rho, d}\right)$ обозначает класс соответствующих функций.

ОПРЕДЕЛЕНИЕ 2. Пусть $C_{1}$ и $C_{2}-$ классы интегрируемых в каком-то смысле функций. Скажем, что $C_{1} \subset C_{2}$, если из $f \in C_{1}$ следует $f \in C_{2}$ и

$$
\left(C_{1}\right) \int f=\left(C_{2}\right) \int f .
$$

Равенство $C_{1}=C_{2}$ означает, что $C_{1} \subset C_{2}$ и $C_{2} \subset C_{1}$. Если выполняется включение $C_{1} \subset C_{2}$, будем говорить, что $\left(C_{2}\right)$-интеграл покрывает $\left(C_{1}\right)$-интеграл.

Несложно показать, что для всех $\rho \in(0,1]$ выполнены включения

$$
P \subset P_{\rho} \subset P_{\rho, d}
$$

ОПРЕДЕЛЕНИЕ 3 . Пусть $C_{1}$ и $C_{2}$ - классы интегрируемых в каком-то смысле функций. Соответствующие интегралы называются непротиворечивыми, если

$$
\left(C_{1}\right) \int f=\left(C_{2}\right) \int f \quad \text { для всех } f \in C_{1} \cap C_{2} .
$$

Далее $B(\mathbf{z}, r)$ обозначает шар радиуса $r$ с центром в точке $\mathbf{z}$.

ОПРеДЕЛЕНИЕ 4. Пусть задано $\rho \in(0,1]$. Разбиением сегмента $I_{0} \in \mathscr{B}_{\rho}$ называется набор $P=\left\{\left(\mathbf{z}_{j}, I_{j}\right)\right\}_{j=1}^{m}$, где $\mathbf{z}_{j} \in I_{0}, I_{j}$ - попарно неперекрывающиеся сегменты из $\mathscr{B}_{\rho}, \bigcup_{j=1}^{m} I_{j}=I_{0}$. Если же $\bigcup_{j=1}^{m} I_{j}$ строго лежит в $I_{0}$, то 
набор $P$ называется частичным разбиением сегмента $I_{0}$. Если задана функция $\delta(\mathbf{z}): I_{0} \rightarrow(0 ;+\infty)$, то разбиение $P$ (частичное разбиение $P$ ) называется $(\delta(\mathbf{z}), \rho)$-согласованным (или просто $(\delta(\mathbf{z}), \rho)$-разбиением), когда $\mathbf{z}_{j} \in I_{j} \subset$ $B\left(\mathbf{z}_{j}, \delta\left(\mathbf{z}_{j}\right)\right)$ и $\operatorname{reg}\left(I_{j}\right) \geqslant \rho$.

ОПРеДЕЛЕНиЕ 5. Пусть $\rho \in(0,1)$. Функция $f:[0,1]^{M} \rightarrow \mathbb{R}$ интегрируема по Хенстоку в $\rho$-регулярном смысле, если существует действительное $A$ и для любого $\varepsilon>0$ существует функция $\delta(\mathbf{z})>0$ такая, что для всех $(\delta(\mathbf{z}), \rho)$ разбиений $P=\left\{\left(\mathbf{z}_{j} ; I_{j}\right)\right\}_{j=1}^{m}$ выполняется неравенство

$$
\left|\sum_{j=1}^{m} f\left(\mathbf{z}_{j}\right)\right| I_{j}|-A|<\varepsilon .
$$

Число $A$ назовем $H_{\rho}$-интегралом.

Класс функций, интегрируемых в указанном смысле, обозначим символом $H_{\rho}$. Заменив в определениях 4 и $5 \mathscr{B}_{\rho}$ на $\mathscr{B} \rho, d$, получим определение двоччного $\rho$-регулярного интеграла Хенстока. Класс соответствующих функций обозначим символом $H_{\rho, d}$. Очевидно, $H_{\rho} \subset H_{\rho, d}$.

Отметим ряд свойств $\left(H_{\rho, d}\right)$-интеграла, которые следуют из [9]. Число $A$ из определения 5 определяется единственным образом. Далее, если функция $f(\mathbf{z})$ является $\left(H_{\rho, d}\right)$-интегрируемой на $[0,1]^{M}$, то она является $\left(H_{\rho, d}\right)$ интегрируемой и на любом двоичном интервале из $[0,1]^{M}$. Наконец, неопределенный $\left(H_{\rho, d}\right)$-интеграл, т.е. функция $F(\Delta)=\left(H_{\rho, d}\right) \int_{\Delta} f(\mathbf{z}) d \mathbf{z}$, является функцией из класса $A_{\mathscr{B}}{ }_{\rho, d}$.

Связь между интегралами Перрона и Хенстока выражена в следующем утверждении (см. [9; с. 19]).

Teopema A. $P_{\rho}=H_{\rho}, P_{\rho, d}=H_{\rho, d}$.

Отметим, что интегралы Хенстока могут быть определены лишь для всюду конечных функций, а интегралы Перрона - и для почти всюду конечных функций. Поэтому в утверждении теоремы А нужно считать классы интегрируемых функций состоящими лишь из всюду конечных функций. Однако значения интегралов Перрона и Хенстока не изменятся, если функцию изменить на множестве нулевой меры, так что можно считать, что все рассматриваемые функции всюду конечны.

ЗАмЕчАНиЕ 1. В дальнейшем нам понадобится интегрировать функции, которые не определены на множестве $S$ меры нуль. В этом случае, рассматривая любой из перечисленных выше интегралов от этих функций, мы считаем эти функции доопределенными на множестве $S$ любыми конечными значениями. При этом значения интегралов от этих функций не зависят от того, как именно мы доопределим эти функции конечными значениями на множестве $S$.

Дадим характеризацию $\left(H_{\rho, d}\right)$-интегралу, т.е. найдем такие необходимые и достаточные условия на функцию множества $F(\Delta)$, чтобы та являлась неопределенным $\left(H_{\rho, d}\right)$-интегралом некоторой функции $f(\mathbf{z})$. Для многих обобщений 
интеграла Лебега характеризация часто дается в терминах некоторой вариационной меры, порожденной функцией множества. До конца этого параграфа будем считать, что у нас выбрано и зафиксировано произвольное $\rho \in(0,1]$.

ОПРЕДЕЛЕНИЕ 6 . Пусть $I_{j} \in \mathscr{B}_{\rho, d}(j=1, \ldots, p)$. Скажем, что частичное разбиение $P=\left\{\left(\mathbf{z}_{j}, I_{j}\right)\right\}_{j=1}^{p}$ связано с множеством $E \subset[0,1]^{M}$, если $\mathbf{z}_{j} \in E$ для всех $j=1, \ldots, p$. Пусть заданы функции $F(\Delta): \mathscr{B}_{\rho, d} \rightarrow \mathbb{R}$ и $\delta(\mathbf{z}): E \rightarrow$ $(0,+\infty)$; тогда положим

$$
\operatorname{Var}^{\rho, d}(F, \delta, E)=\sup \sum_{j}\left|F\left(I_{j}\right)\right|,
$$

где "супремум" берется по всем $(\delta(\mathbf{z}), \rho)$-разбиениям, связанным с множеством $E$. Определим $\rho$-регулярную двоичную вариационную меру множества $E$, порожденную функцией $F$, равенством

$$
V_{F}^{\rho, d}(E)=\inf _{\delta} \operatorname{Var}^{\rho, d}(F, \delta, E) .
$$

ОПРедЕЛЕниЕ 7. Вариационная мера $V_{F}^{\rho, d}$ называется абсолютно непреръвной на множестве $E \subset[0,1]^{M}$, если $V_{F}^{\rho, d}(C)=0$ для всех множеств $C \subset E$ c mes $C=0$.

Нам понадобятся результаты работы [10]. Там рассматривался двумерный случай, однако доказательства всех утверждений этой работы автоматически переносятся на случай произвольной конечной размерности. В [10] была доказана

Теорема В. Если $F(\Delta)=\left(H_{\rho, d}\right) \int_{\Delta} f(\mathbf{z}) d \mathbf{z}$, то п.в. на $[0,1]^{M}$ выполнено равенство $D_{\mathscr{B}}{ }_{\rho, d} F(\mathbf{z})=f(\mathbf{z})$.

Из теоремы В и утверждений 2 и 3 из работы [11] вытекает

ТЕОрема 1. Функиия $F \in A_{\mathscr{B}_{\rho, d}}$ есть неопределенный $\left(H_{\rho, d}\right)$-интеграл от некоторой функции $f(\mathbf{z}):[0,1]^{M} \rightarrow \mathbb{R}$ тогда и толъко тогда, когда $F$ порождает абсолютно непрерывную на $[0,1]^{M}$ вариационную меру $V_{F}^{\rho, d}$.

Теоремы такого рода называются теоремами о полной характеризации интеграла. Аналогичная теорема для интеграла Хенстока-Курцвейля была установлена в [12], а для ряда многомерных обобщенных интегралов - в работах [13]-[15] и др.

Лемма 1. Пустъ $f(\mathbf{z}) \in H_{\rho, d} u F(\Delta)=\left(H_{\rho, d}\right) \int_{\Delta} f(\mathbf{z}) d \mathbf{z}$. Тогда для любой точки $\mathbf{z} \in[0,1]^{M}$ выполнено условие

$$
\lim F(\Delta)=0, \quad \mathbf{z} \in \Delta, \quad|\Delta| \rightarrow 0, \quad \Delta-\text { двоичный интервал } с \operatorname{reg}(\Delta) \geqslant \rho \text {. }
$$

ДокАзАтЕльство. Утверждение леммы сразу вытекает из известного свойства Хаке для многомерных интегралов хенстоковского типа (см. [16; теорема 3.3]). 


\section{§4. Об одном интеграле перроновского типа}

В этом параграфе мы рассмотрим интегралы, помогающие решать проблемы восстановления коэффициентов рядов (1). Пусть $\Sigma$ обозначает множество двумерных векторов $\boldsymbol{\sigma}=\left(\sigma_{1}, \sigma_{2}\right)$, состоящих из нулей и единиц. Далее, пусть заданы точка $\mathbf{z} \in[0,1]^{2}$ и любая $(\mathbf{z})$-последовательность $\left\{\Delta_{k_{1}, k_{2}}\right\}$. Обозначим $\Delta_{k_{j}}^{0}=\Delta_{k_{j}}, \Delta_{k_{j}}^{1}=\Delta_{k_{j}-1} \backslash \Delta_{k_{j}}$. Тогда положим

$$
\begin{array}{ll}
\Delta_{k_{1}, k_{2}}^{1}=\Delta_{k_{1}}^{0} \times \Delta_{k_{2}}^{0}, & \Delta_{k_{1}, k_{2}}^{2}=\Delta_{k_{1}}^{0} \times \Delta_{k_{2}}^{1}, \\
\Delta_{k_{1}, k_{2}}^{3}=\Delta_{k_{1}}^{1} \times \Delta_{k_{2}}^{1}, & \Delta_{k_{1}, k_{2}}^{4}=\Delta_{k_{1}}^{1} \times \Delta_{k_{2}}^{0} .
\end{array}
$$

Очевидно, $\Delta_{k_{1}, k_{2}}^{i}$ есть двоичный интервал ранга $\left(k_{1}, k_{2}\right)$, а набор $\left\{\Delta_{k_{1}, k_{2}}^{i}: i \in\right.$ $\{1,2,3,4\}\}$ состоит из четырех попарно неперекрывающихся двоичных интервалов, составляющих в объединении двоичный интервал ранга $\left(k_{1}-1, k_{2}-1\right)$. Пусть выражение $|\boldsymbol{\sigma}|$ обозначает $\left|\sigma_{1}\right|+\left|\sigma_{2}\right|$. Нам потребуется следующий результат.

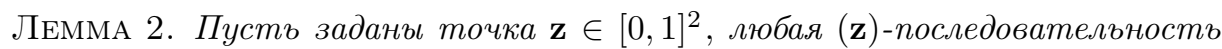
$\left\{\Delta_{\mathbf{k}}\right\}$ и функиия множества $\Phi \in A_{\mathscr{B}_{1 / 2, d}}$. Тогда для любого $i \in\{1,2,3,4\}$ имеет место неравенство

$$
\left|\Phi\left(\Delta_{n, n}^{i}\right)\right| \leqslant \sum_{\boldsymbol{\sigma}=\left(\sigma_{1}, \sigma_{2}\right) \in \Sigma}\left|\Phi\left(\Delta_{n-\sigma_{1}, n-\sigma_{2}}\right)\right| .
$$

ДокАЗАтЕЛЬСтво. В условиях леммы имеют место соотношения

$$
\begin{aligned}
\Phi\left(\Delta_{n, n}^{2}\right)= & \left(\Phi\left(\Delta_{n, n}^{1}\right)+\Phi\left(\Delta_{n, n}^{2}\right)\right)-\Phi\left(\Delta_{n, n}^{1}\right)=\Phi\left(\Delta_{n, n-1}\right)-\Phi\left(\Delta_{n, n}\right) \\
\Phi\left(\Delta_{n, n}^{4}\right)= & \left(\Phi\left(\Delta_{n, n}^{1}\right)+\Phi\left(\Delta_{n, n}^{4}\right)\right)-\Phi\left(\Delta_{n, n}^{1}\right)=\Phi\left(\Delta_{n-1, n}\right)-\Phi\left(\Delta_{n, n}\right) \\
\Phi\left(\Delta_{n, n}^{3}\right)= & \left(\Phi\left(\Delta_{n, n}^{1}\right)+\Phi\left(\Delta_{n, n}^{2}\right)+\Phi\left(\Delta_{n, n}^{3}\right)+\Phi\left(\Delta_{n, n}^{4}\right)\right) \\
& -\left(\Phi\left(\Delta_{n, n}^{1}\right)+\Phi\left(\Delta_{n, n}^{2}\right)\right)-\left(\Phi\left(\Delta_{n, n}^{1}\right)+\Phi\left(\Delta_{n, n}^{4}\right)\right)+\Phi\left(\Delta_{n, n}^{1}\right) \\
= & \Phi\left(\Delta_{n-1, n-1}\right)-\Phi\left(\Delta_{n, n-1}\right)-\Phi\left(\Delta_{n-1, n}\right)+\Phi\left(\Delta_{n, n}\right) .
\end{aligned}
$$

Из соотношения (6) следует выполнение неравенства (5) для квадрата $\Delta_{n, n}^{2}$, из $(7)$ - для квадрата $\Delta_{n, n}^{4}$, а из $(8)$ - для квадрата $\Delta_{n, n}^{3}$. Выполнение неравенства (5) для квадрата $\Delta_{n, n}^{1}$ очевидно. Лемма доказана.

Рассмотрим двумерный случай одного $M$-мерного обобщенного интеграла, введенного в [6].

ОПРЕДЕЛЕниЕ 8. Пусть заданы $\rho \in(0,1 / 2]$ и не более чем счетное множество $W \subset[0,1]^{2}$. Пусть на квадрате $[0,1]^{2}$ или по крайней мере в точках множества $\left(I_{d}\right)^{2} \backslash W$ определена конечная функция $f(\mathbf{z})$.

Скажем, что функция $f(\mathbf{z})$ является $\left(P_{d}^{\rho, *}\right)$-интегрируемой на $[0,1]^{2}$, если для любого $\varepsilon>0$ найдутся мажоранта $F_{1} \in \bar{A}_{\mathscr{B}_{\rho, d}}$ и миноранта $F_{2} \in \underline{A}_{\mathscr{B}_{\rho, d}}$ со следующими свойствами:

1) $\underline{D}_{\mathscr{B}_{\rho, d}} F_{1}(\mathbf{z}) \geqslant f(\mathbf{z}) \geqslant \bar{D}_{\mathscr{B}_{\rho, d}} F_{2}(\mathbf{z})$ при $\mathbf{z} \in\left(I_{d} \times I_{d}\right) \backslash W$; 
2) если $\mathbf{z} \in\left(Q_{d} \times I_{d}\right) \backslash W$ (или $\left.\mathbf{z} \in\left(I_{d} \times Q_{d}\right) \backslash W\right)$, то для любой $(\mathbf{z})$-последовательности $\left\{\Delta_{k, l}\right\}$ при $j=1,2$ выполнено условие

$$
\begin{gathered}
\lim _{\substack{\min \{k, l\} \rightarrow \infty \\
\operatorname{reg}\left(\Delta_{k, l}\right) \geqslant 2 \rho}} \frac{1}{\sqrt{\left|\Delta_{k, l}\right|}}\left(F_{j}\left(\Delta_{k, l}^{1}\right)-F_{j}\left(\Delta_{k, l}^{4}\right)\right)=0 \\
\left(\lim _{\substack{\min \{k, l\} \rightarrow \infty \\
\operatorname{reg}\left(\Delta_{k, l}\right) \geqslant 2 \rho}} \frac{1}{\sqrt{\left|\Delta_{k, l}\right|}}\left(F_{j}\left(\Delta_{k, l}^{1}\right)-F_{j}\left(\Delta_{k, l}^{2}\right)\right)=0\right)
\end{gathered}
$$

$3)$ для $j=1,2$, для каждой точки $\mathbf{z} \in[0,1]^{2}$ и каждой $(\mathbf{z}, \square)$-последовательности $\left\{\Delta_{n}\right\}$ выполнено равенство

$$
\lim _{n \rightarrow \infty}\left[F_{j}\left(\Delta_{n}^{1}\right)-F_{j}\left(\Delta_{n}^{2}\right)+F_{j}\left(\Delta_{n}^{3}\right)-F_{j}\left(\Delta_{n}^{4}\right)\right]=0
$$

4) $F_{1}\left([0,1]^{2}\right)-F_{2}\left([0,1]^{2}\right)<\varepsilon$.

$\left(P_{d}^{\rho, *}\right)$-интеграл от функции $f(\mathbf{z})$ по любому $\rho$-регулярному двоичному интервалу $\Delta$ определяется формулой

$$
\left(P_{d}^{\rho, *}\right) \int_{\Delta} f(\mathbf{z}) d \mathbf{z}=\inf _{F_{1}} F_{1}(\Delta)=\sup _{F_{2}} F_{2}(\Delta) .
$$

По аддитивности понятие $\left(P_{d}^{\rho, *}\right)$-интеграла можно распространить на любой двоичный интервал.

Следующая теорема есть теорема 6 из [6], сформулированная для случая $M=2$.

Теорема С. Пусть заданы $\rho \in(0,1 / 2]$ и не более чем счетное множество W. Предположим, что ряд (1) всюду на $[0,1]^{2}$ удовлетворяет одному из следующих условий:

$$
\begin{aligned}
& a_{n, m} \chi_{n, m}(\mathbf{z})=o_{\mathbf{z}}(n m), \quad \min \{n, m\} \rightarrow \infty, \quad \operatorname{reg}((n, m)) \geqslant 2 \rho ; \\
& S_{N, M}(\mathbf{z})=o_{\mathbf{z}}(N M), \quad \min \{N, M\} \rightarrow \infty, \quad \operatorname{reg}((N, M)) \geqslant \rho .
\end{aligned}
$$

Пусть этот ряд во всех точках множества $\left(I_{d} \times I_{d}\right) \backslash W$ сходится $\rho$-регулярно или по прямоугольникам $\kappa$ конечной функции $f(\mathbf{z})$, а во всех точках $\mathbf{z} \in[0,1]^{2} \backslash W$, имеющих ровно одну $Q_{d}$-координату, для частичных сумм этого ряда выполняется условие

$$
S_{N, M}(\mathbf{z})=o_{\mathbf{z}}(\sqrt{N M}), \quad \min \{N, M\} \rightarrow \infty, \quad \operatorname{reg}((N, M)) \geqslant \rho .
$$

Тогда функиия $f(\mathbf{z})$ является $\left(P_{d}^{\rho, *}\right)$-интегрируемой, а ряд (1) является рядом Фуръе функции $f(\mathbf{z})$ относителъно $\left(P_{d}^{\rho, *}\right)$-интеграла.

Следующий результат обобщает теоремы 1 из [17]-[19], а при $M=2$ - и теорему 3 из [6].

Теорема 2. Пусть заданы не более чем счетное множество $W$ и функиия $\Phi(\Delta) \in \bar{A}_{\mathscr{B}_{1 / 2, d}}$, причем в каждой точке $\mathbf{z} \in[0,1]^{2}$ для функиии $\Phi$ и для всех $(\mathbf{z}, \square)$-последовательностей $\left\{\Delta_{n}\right\}$ справедливо равенство (11). 
Предположим, что выполнены следующие условия:

1) если $\mathbf{z} \in\left(I_{d} \times I_{d}\right) \backslash W$, то для $(\mathbf{z}, \square)$-последовательности $\left\{\Delta_{n}\right\}$, а также для некоторой возрастающей последовательности натуральных чисел $n_{l}=n_{l}(\mathbf{z})$ выполнено соотношение

$$
\varliminf_{l \rightarrow \infty} \frac{\Phi\left(\Delta_{n_{l}}\right)}{\left|\Delta_{n_{l}}\right|} \geqslant 0 ;
$$

2) если $\mathbf{z} \in\left(Q_{d} \times I_{d}\right) \backslash W\left(\right.$ или $\left.\mathbf{z} \in\left(I_{d} \times Q_{d}\right) \backslash W\right)$, то для любой $(\mathbf{z}, \square)$ последовательности $\left\{\Delta_{n}\right\}$ и для некоторой возрастающей последовательности натуральных чисел $n_{l}$, зависящей от $\mathbf{z} u\left\{\Delta_{n}\right\}$, выполнено условие

$$
\begin{aligned}
& \lim _{l \rightarrow \infty} \frac{1}{\sqrt{\left|\Delta_{n_{l}}\right|}}\left(\Phi\left(\Delta_{n_{l}}^{1}\right)-\Phi\left(\Delta_{n_{l}}^{4}\right)\right)=0 \\
& \left(\lim _{l \rightarrow \infty} \frac{1}{\sqrt{\left|\Delta_{n_{l}}\right|}}\left(\Phi\left(\Delta_{n_{l}}^{1}\right)-\Phi\left(\Delta_{n_{l}}^{2}\right)\right)=0\right) .
\end{aligned}
$$

Тогда $\Phi(I) \geqslant 0$ для любого двоичного интервала $I \in \mathscr{B}_{1 / 2, d}$.

ДокАЗАТЕЛЬство. Предположим, что утверждение теоремы не является верным и $\Phi\left(I_{1}\right)<0$ для некоторого двоичного интервала $I_{1} \in \mathscr{B}_{1 / 2, d}$. Так как $\Phi \in \bar{A}_{\mathscr{B}_{1 / 2, d}}$, то, разбив интервал $I_{1}$ на конечное число двоичных квадратов, получим, что $\Phi\left(I_{0}\right)<0$ для некоторого двоичного квадрата $I_{0} \subset I_{1}$. Введем новую функцию $\Phi_{0}(\Delta)=\Phi(\Delta)+\varepsilon|\Delta|$, где $\varepsilon>0$ настолько мало, что $\Phi_{0}\left(I_{0}\right)<0$. Очевидно, $\Phi_{0} \in \bar{A}_{\mathscr{B}_{1 / 2, d}}$. Пусть задана некоторая функция множества $\widetilde{\Phi}$. Назовем двоичный интервал $I \subset I_{0} \widetilde{\Phi}$-правильным, если $\widetilde{\Phi}(I)<0$. Следующая лемма была доказана в [6].

Лемма А. Пусть для функиии $\widetilde{\Phi} \in \bar{A}_{\mathscr{B}_{1 / 2, d}}$ в каждой точке $\mathbf{z} \in[0,1]^{2}$ въполнено условие (11) и непекрывающиеся $\widetilde{\Phi}$-правильные двоччные квадраты $I^{1}$ и $I^{2}$, лежащие в $I$.

Назовем $\widetilde{\Phi}$-цепъю вложенный конечный или счетный набор $\left\{\Delta_{n}\right\}_{n=n_{1}}^{n_{2}}\left(n_{2}\right.$ может равняться $\infty) \widetilde{\Phi}$-правильных двоичных квадратов таких, что ранг квадрата $\Delta_{n}$ равен $n$. Скажем, что конечная цепь $\left\{\Delta_{n}\right\}_{n=n_{1}}^{n_{2}}$ cвязывает два двоичных квадрата, $J_{1}$ и $J_{2}$, если $J_{i}=\Delta_{n_{i}}$ при $i=1,2$. Из доказательства леммы А в [6] следует, что квадраты $I^{1}$ и $I^{2}$ из данной леммы можно выбрать связывающимися $\widetilde{\Phi}$-цепью с квадратом $I$.

Следуя [6], назовем типом двоичного интервала (2) пару чисел $\left(u_{1}, u_{2}\right)$ таких, что $u_{i}=p_{i}(\bmod 2)$. Отметим, что если $\left\{\Delta_{n}\right\}$ является $(\mathbf{z}, \square)$-последовательностью для некоторой точки $\mathbf{z}=\left(z_{1}, z_{2}\right)$ и $\left(i_{1}^{n}, i_{2}^{n}\right)$ - тип квадрата $\Delta_{n}$, то $z_{j} \in Q_{d}$ тогда и только тогда, когда в последовательности $i_{j}^{n}$ одно из чисел 0 или 1 встречается лишь конечное число раз.

Дальнейшее доказательство напоминает доказательство теоремы 3 из [6] и использует возможность реализации одного из двух логически возможных случаев. 
Случай А. Для любого номера $k \in\{1,2\}$, любого $u \in\{0,1\}$ и любого правильного двоичного квадрата $I^{\prime} \subset I_{0}$ существует $\Phi_{0}$-правильный двоичный квадрат $I^{\prime \prime} \subset I^{\prime}$, который можно связать $\Phi_{0}$-цепью с $I^{\prime}$, причем в типе квадрата $I^{\prime \prime}$ на $k$-м месте стоит число $u$.

Случай В. Существуют $k_{1} \in\{1,2\}, u_{1} \in\{0,1\}$ и $\Phi_{0}$-правильный двоичный квадрат $I^{\prime}$, который не связывается $\Phi_{0}$-цепью ни с одним $\Phi_{0^{-}}$ правильным двоичным квадратом $I^{\prime \prime} \subset I^{\prime}$, в типе которого на $k_{1}$-м месте стоит число $u_{1}+1(\bmod 2)$.

Пусть имеет место случай А. Построим индукцией по $n$ набор двоичных кубов $I_{s}^{j}, s=0,1, \ldots, j=0, \ldots, 2^{s}-1$, следующим образом. Пусть $I_{0}^{0}=$ $I_{0}$. Предположим, что мы построили искомый набор для $s \leqslant S$. Пользуясь условиями случая А, при каждом $j=1, \ldots, 2^{S}-1$ для квадрата $I_{S}^{j}$ построим $\Phi_{0}$-правильные двоичные квадраты $M_{S}^{j} \subset L_{S}^{j} \subset K_{S}^{j} \subset J_{S}^{j} \subset I_{S}^{j}$, любые два из которых связываются $\Phi_{0}$-цепью и такие, что в типе квадратов $M_{S}^{j}$ (или $\left.L_{S}^{j}\right)$ на первом месте стоит 0 (или 1 ), а в типе квадратов $K_{S}^{j}$ (или $J_{S}^{j}$ ) на втором месте стоит 0 (или 1). Пользуясь леммой А, найдем $\Phi_{0}$-правильные двоичные квадраты $I_{S+1}^{2 j}$ и $I_{S+1}^{2 j+1}$, лежащие в квадрате $M_{S}^{j}$ и соединяющиеся с ним $\Phi_{0^{-}}$ цепью. Таким образом, мы построили искомый набор при $s=S+1$. По построению набор $I_{s}^{j}$ обладает тем свойством, что множество

$$
W_{A}=\bigcap_{s=0}^{\infty} \bigcup_{j=0}^{2^{s}-1} I_{s}^{j}
$$

лежит во множестве $\left(I_{d}\right)^{2}$, причем к каждой точке $\mathbf{z} \in W_{A}$ стягивается бесконечная $\Phi_{0}$-цепь $\left\{\Delta_{n}\right\}_{n=n_{0}}^{\infty}\left(n_{0}\right.$ есть ранг квадрата $\left.I_{0}^{0}\right)$. Кроме того, множество $W_{A}$ непусто и совершенно. Возьмем любую точку $\mathbf{z} \in W_{A}$ и $(\mathbf{z}, \square)$ последовательность $\left\{\Delta_{n}\right\}$. Тогда для любой возрастающей последовательности натуральных чисел $n_{l}$

$$
\varlimsup_{l \rightarrow \infty} \frac{\Phi\left(\Delta_{n_{l}}\right)}{\left|\Delta_{n_{l}}\right|}=\varlimsup_{l \rightarrow \infty} \frac{\Phi_{0}\left(\Delta_{n_{l}}\right)}{\left|\Delta_{n_{l}}\right|}-\varepsilon \leqslant 0-\varepsilon=-\varepsilon<0,
$$

откуда согласно условию 1$)$ теоремы $2 W_{A} \subset W$. Но множество $W$ не более чем счетно, а $W_{A}$ - несчетно. Противоречие доказывает теорему в случае А.

Пусть имеет место случай В. Без ограничения общности считаем, что $k_{1}=1$, $u_{1}=0$. Пусть ранг квадрата $I^{\prime}$ равен $n_{1}$. Рассмотрим любую бесконечную $\Phi_{0^{-}}$ цепь $\left\{\Delta_{n}\right\}_{n=n_{1}}^{\infty}$, начинающуюся с квадрата $I^{\prime}$. По условию случая В в типе каждого квадрата из этой цепи на первом месте стоит нуль. Ясно, что в этом случае такая цепь стягивается к точке $\mathbf{z}_{0} \in X$, где $X$ есть левая четверть границы квадрата $I^{\prime}$. Далее, пусть данная цепь дополнена до $\left(\mathbf{z}_{0}\right)$-последовательности $\left\{\Delta_{k, l}\right\}$. Тогда из условия случая В вытекает, что при $n \geqslant n_{1}+1$ двоичный куб $\Delta_{n-1, n} \backslash \Delta_{n, n}$ не является $\Phi_{0}$-правильным, т.е.

$$
\Phi_{0}\left(\Delta_{n-1, n} \backslash \Delta_{n, n}\right) \geqslant 0 .
$$

На двоичных кубах $\Delta \subset \Delta^{\prime}$ рассмотрим функцию

$$
\Phi_{2}(\Delta)=\Phi_{0}(\Delta)+\Phi_{1}(\Delta), \quad \text { где } \Phi_{1}(\Delta)=\varepsilon_{1} \operatorname{mes}_{1}(\Delta \cap X) .
$$


Здесь $\operatorname{mes}_{1}(\cdot)$ означает линейную меру множества, а $\varepsilon_{1}>0$ настолько мало, что $\Phi_{2}\left(\Delta^{\prime}\right)<0$. Из геометрических соображений ясно, что функция $\Phi_{1}$ аддитивна, следовательно, $\Phi_{1}, \Phi_{2} \in \bar{A}_{\mathscr{B}_{1 / 2, d}}$. Кроме того, для функций $\Phi_{0}$ и $\Phi_{1}$ выполнено условие (11), откуда следует, что и для функции $\Phi_{2}$ выполняется это условие. Рассмотрим множество

$$
W_{B}=\bigcap_{n=n_{1}}^{\infty} \bigcup_{J \subset \Delta^{\prime}} J, \quad \text { где } J-\Phi_{2} \text {-правильный двоичный квадрат ранга } n \text {. }
$$

$\mathrm{K}$ каждой точке множества $\mathbf{z} \in W_{B}$ стягивается $\Phi_{2}$-цепь $\left\{\Delta_{n}\right\}_{n=n_{1}}^{\infty}$. Поскольку $\Phi_{0}(\Delta) \leqslant \Phi_{2}(\Delta)$, то $\left\{\Delta_{n}\right\}_{n=n_{1}}^{\infty}-$ также $\Phi_{0}$-цепь. Как отмечалось выше, в этом случае $\mathbf{z} \in X$, откуда в силу произвольности $\mathbf{z} \in W_{B} W_{B} \subset X$. Кроме того, функция $\Phi_{2}$ удовлетворяет условию (11), поэтому в силу леммы А множество $W_{B}$ содержит непустое совершенное подмножество. Так как $W_{B} \subset X$, а $X$ есть левая часть границы двоичного квадрата, то, выкинув из множества $W_{B}$ точки с двумя $Q_{d}$-координатами, получим несчетное множество $W_{B}^{\prime}=$ $W_{B} \cap\left(Q_{d} \times I_{d}\right)$.

Возьмем любую точку $\mathbf{z}_{0} \in W_{B}^{\prime}$ и покажем, что для нее не выполняется условие (16). $\mathrm{K}$ точке $\mathbf{z}_{0}$ стягивается $\Phi_{2}$-цепь $\left\{\Delta_{n}\right\}_{n=n_{1}}^{\infty}$. Пусть эта цепь дополнена до $\left(\mathbf{z}_{0}\right)$-последовательности $\left\{\Delta_{k, l}\right\}$. Поскольку $\Phi_{2}(\Delta) \geqslant \Phi_{0}(\Delta)$, то в силу (18) имеет место неравенство $\Phi_{2}\left(\Delta_{n-1, n} \backslash \Delta_{n, n}\right) \geqslant 0$, откуда с учетом неравенства $\Phi_{2}\left(\Delta_{n, n}\right)<0$ получаем

$$
\Phi_{2}\left(\Delta_{n}^{1}\right)-\Phi_{2}\left(\Delta_{n}^{4}\right)=\Phi_{2}\left(\Delta_{n, n}\right)-\Phi_{2}\left(\Delta_{n-1, n} \backslash \Delta_{n, n}\right)<0 .
$$

Рассмотрим следующее соотношение:

$$
\begin{aligned}
\Phi\left(\Delta_{n}^{1}\right)- & \Phi\left(\Delta_{n}^{4}\right)=\left[\Phi_{2}\left(\Delta_{n}^{1}\right)-\Phi_{2}\left(\Delta_{n}^{4}\right)\right] \\
& -\varepsilon\left[\left|\Delta_{n}^{1}\right|-\left|\Delta_{n}^{4}\right|\right]-\varepsilon^{\prime}\left[\operatorname{mes}_{1}\left(\Delta_{n}^{1} \cap X\right)-\operatorname{mes}_{1}\left(\Delta_{n}^{4} \cap X\right)\right] .
\end{aligned}
$$

Согласно (19) выражение в первых скобках в правой части (20) отрицательно. Выражение во вторых скобках, очевидно, равно нулю. Так как $\operatorname{mes}_{1}\left(\Delta_{n}^{1} \cap\right.$ $X)=2^{-n}$, a $\operatorname{mes}_{1}\left(\Delta_{n}^{4} \cap X\right)=0$, то выражение в третьих скобках равно $2^{-n}$. Тогда

$$
\frac{1}{\sqrt{\left|\Delta_{n}\right|}}\left(\Phi\left(\Delta_{n}^{1}\right)-\Phi\left(\Delta_{n}^{4}\right)\right) \leqslant \frac{1}{\sqrt{\left|\Delta_{n}\right|}}\left(0-0-\varepsilon^{\prime} 2^{-n}\right)=-\frac{\varepsilon^{\prime} 2^{-n}}{2^{-n}}=-\varepsilon^{\prime}<0,
$$

т.е. в точке $\mathbf{z} \in W_{B}^{\prime}$ не выполнено условие (16). Поэтому из условий теоремы в силу произвольности $\mathbf{z} \in W_{B}^{\prime}$ вытекает, что $W_{B}^{\prime} \subset W$. Но множество $W$ не более чем счетно, а $W_{B}^{\prime}$ - несчетно. Противоречие доказывает теорему в случае В. Теорема доказана полностью.

Теорема 2 позволяет построить семейство интегралов перроновского типа, каждый из которых покрывает $\left(P_{d}^{\rho, *}\right)$-интеграл.

ОПРеДЕЛЕНИЕ 9. Пусть множество $W \subset[0,1]^{2}$ не более чем счетно, а в точ$\operatorname{kax} \mathbf{z} \in\left(I_{d}\right)^{2} \backslash W$ задана конечная функция $f(\mathbf{z})$. Пусть для каждой точки 
$\mathbf{z} \in[0,1]^{2}$ и каждой $(\mathbf{z})$-последовательности задана возрастающая последовательность натуральных чисел $n_{l}$.

Скажем, что функция $f(\mathbf{z})$ является $\left(P\left(n_{l}\right)\right)$-интегрируемой на $[0,1]^{2}$, если для любого $\varepsilon>0$ найдутся мажоранта $F_{1} \in \bar{A}_{\mathscr{B}_{1 / 2, d}}$ и миноранта $F_{2} \in \underline{A}_{\mathscr{B}_{1 / 2, d}}$ со следующими свойствами:

1) если $\mathbf{z} \in\left(I_{d}\right)^{2} \backslash W$, то для $(\mathbf{z}, \square)$-последовательности $\left\{\Delta_{n}\right\}$ и последовательности $n_{l}=n_{l}(\mathbf{z})$ выполнено равенство

$$
\varliminf_{l \rightarrow \infty} \frac{F_{1}\left(\Delta_{n_{l}}\right)}{\left|\Delta_{n_{l}}\right|} \geqslant f(\mathbf{z}) \geqslant \varlimsup_{l \rightarrow \infty} \frac{F_{2}\left(\Delta_{n_{l}}\right)}{\left|\Delta_{n_{l}}\right|} ;
$$

2) если $\mathbf{z} \in\left(Q_{d} \times I_{d}\right) \backslash W$ (или $\left.\mathbf{z} \in\left(I_{d} \times Q_{d}\right) \backslash W\right)$, то для любой $(\mathbf{z}, \square)$ последовательности $\left\{\Delta_{n}\right\}$, для соответствующей последовательности $n_{l}$, а также функций $F_{1}$ и $F_{2}$ выполнено равенство (16) (или равенство (17)).

$3)$ для каждой точки $\mathbf{z} \in[0,1]^{2}$, для функций $F_{1}$ и $F_{2}$ и каждой $(\mathbf{z}, \square)$ последовательности $\left\{\Delta_{n}\right\}$ выполнено равенство (11);

4) $F_{1}\left([0,1]^{2}\right)-F_{2}\left([0,1]^{2}\right)<\varepsilon$.

$\left(P\left(n_{l}\right)\right)$-интеграл от функции $f(\mathbf{z})$ по любому двоичному интервалу $\Delta \in$ $\mathscr{B}_{1 / 2, d}$ определяется как

$$
\left(P\left(n_{l}\right)\right) \int_{\Delta} f(\mathbf{z}) d \mathbf{z}=\inf _{F_{1}} F_{1}(\Delta)=\sup _{F_{2}} F_{2}(\Delta) .
$$

По аддитивности понятие $\left(P\left(n_{l}\right)\right)$-интеграла можно распространить на любой двоичный интервал.

В дальнейшем набор возрастающих последовательностей $n_{l}$ из определения 9 будем называть допустимым.

ТЕОРЕма 3. При любом допустимом наборе $n_{l}$ и при любом $\rho \in(0,1 / 2]$ $\left(P\left(n_{l}\right)\right)$-интеграл покрывает $\left(P_{d}^{\rho, *}\right)$-интеграл.

ДоказАтельство. Если при $\rho \in(0,1 / 2]$ функции $F_{1} \in \bar{A}_{\mathscr{B}_{\rho, d}}$ и $F_{2} \in \underline{A}_{\mathscr{B}}{ }_{\rho, d}$ удовлетворяют определению $\left(P_{d}^{\rho, *}\right)$-интеграла при некотором $\varepsilon>0$, то они удовлетворяют и определению $\left(P\left(n_{l}\right)\right)$-интеграла при том же $\varepsilon$. Действительно, в этом случае $F_{1} \in \bar{A}_{\mathscr{B}_{1 / 2, d}}, F_{2} \in \underline{A}_{\mathscr{B}_{1 / 2, d}}$. Далее, из того, что $\underline{D}_{\mathscr{B}_{\rho, d}} F_{1}(\mathbf{z}) \geqslant$ $f(\mathbf{z}) \geqslant \bar{D}_{\mathscr{B} \rho, d} F_{2}(\mathbf{z})$, вытекает выполнение условия (21). Так как для функций $F_{1}$ и $F_{2}$ справедливы равенства $(9)$ и $(10)$, то для них справедливы равенства (16) и (17). Из указанных фактов вытекает справедливость теоремы.

Отметим, что определение $\left(P\left(n_{l}\right)\right)$-интеграла не дает ответа на вопрос, зависит ли значение $\left(P\left(n_{l}\right)\right)$-интеграла от выбора последовательностей $n_{l}$. Но если $f \in P_{d}^{\rho, *}$, то в силу теоремы $3 f \in P\left(n_{l}\right)$ для любого допустимого набора $n_{l}$ и

$$
\left(P_{d}^{\rho, *}\right) \int_{[0,1]^{2}} f(\mathbf{z}) d \mathbf{z}=\left(P\left(n_{l}\right)\right) \int_{[0,1]^{2}} f(\mathbf{z}) d \mathbf{z} .
$$




\section{§5. Непротиворечивость двух интегралов перроновского типа}

В этом параграфе мы докажем непротиворечивость при всех $\rho \in(0,1 / 2]$ $\left(P_{d}^{\rho, *}\right)$-интеграла и $\left(H_{1 / 2, d}\right)$-интеграла. Мы используем семейство $\left(P\left(n_{l}\right)\right)$-интегралов, построенных в $\S 4$. По теореме $3\left(P_{d}^{\rho, *}\right)$-интеграл покрывается каждым интегралом из семейства $\left(P\left(n_{l}\right)\right)$-интегралов. Сейчас мы покажем, что семейство $\left(P\left(n_{l}\right)\right)$-интегралов покрывает в некотором смысле и $\left(H_{1 / 2, d}\right)$-интеграл.

ТЕОрема 4. Пусть конечная функиия $f(\mathbf{z})$ определена на $[0,1]^{2}$, кроме, быть может, некоторого множества $S$ меры нуль, на котором ее можно доопределить любыми конечными значениями (см. замечание 1). Если функиия $f(\mathbf{z})$ является $\left(H_{1 / 2, d}\right)$-интегрируемой, то она является $\left(P\left(n_{l}\right)\right)$-интегрируемой для некоторого допустимого набора $n_{l}$, причем соответствующие интегральь совпадают.

ДокАЗАтЕЛЬСтво. Пусть

$$
F(\Delta)=\left(H_{1 / 2, d}\right) \int_{\Delta} f(\mathbf{z}) d \mathbf{z} .
$$

По теореме 1 вариационная мера $V_{F}^{1 / 2, d}$, порожденная функцией $F(\Delta)$, абсолютно непрерывна. Покажем, что $F(\Delta)$ - неопределенный $\left(P\left(n_{l}\right)\right)$-интеграл для некоторого допустимого набора $n_{l}$.

Рассмотрим всевозможные отрезки

$$
\Lambda=\left\{\mathbf{z} \in[0,1]^{2}: z_{s}=a \in Q_{d}\right\} .
$$

Очевидно, имеется счетный набор таких отрезков, и мы можем их занумеровать, обозначив символами $A_{\alpha}, \alpha=1,2, \ldots$. Пусть $B_{l}=\bigcup_{\alpha=1}^{l} A_{\alpha}$. Очевидно, $B_{l} \subseteq B_{l+1}$ для всех $l$ и

$$
\bigcup_{l=1}^{\infty} B_{l}=[0,1]^{2} \backslash\left(I_{d}\right)^{2} .
$$

Все множества $B_{l}$ имеют меру нуль. Воспользовавшись абсолютной непрерывностью вариационной меры $V_{F}^{1 / 2, d}$, индукционно построим последовательность $P_{l}=\left\{\left(\mathbf{z}_{l}^{j}, I_{l}^{j}\right)\right\}_{j=1}^{q_{l}}$ частичных разбиений квадрата $[0,1]^{2}$, связанных с множествами $B_{l}$, а также числовую последовательность $\varepsilon_{l}>0$. Потребуем, чтобы эти последовательности удовлетворяли следующим условиям:

I) все $I_{l}^{j}$ - двоичные квадраты;

II) пусть $s_{l}$ и $t_{l}$ - минимальный и максимальный из рангов квадратов $\left\{I_{l}^{j}\right.$ : $\left.j=1, \ldots, q_{l}\right\}$, тогда $t_{l+1} \geqslant s_{l+1} \geqslant t_{l}+2 ;$

III) последовательность $\varepsilon_{l}>0$ такова, что $\varepsilon_{1}=1$, ряд $\sum_{l=1}^{\infty} \varepsilon_{l}$ сходится и

$$
\sum_{\alpha=l+1}^{\infty} \varepsilon_{\alpha}=o\left(\frac{1}{4^{t_{l}}}\right) \quad \text { при } l \rightarrow \infty ;
$$

IV) пусть по последовательности $\varepsilon_{l}>0$ выбраны функции $\delta_{l}(\mathbf{z}): B_{l} \rightarrow(0,+\infty)$ так, как это делается в определении вариационной меры $V_{F}^{1 / 2, d}$ (см. определение 6$)$, т.е. так, что для любого $\left(\delta_{l}(\mathbf{z}), 1 / 2\right)$-согласованного разбиения 
$P_{l}^{\prime}=\left\{\left(\left(\mathbf{z}_{l}^{j}\right)^{\prime},\left(I_{l}^{j}\right)^{\prime}\right)\right\}_{j=1}^{q_{l}^{\prime}}$, связанного со множеством $B_{l}$, будет выполнено неравенство

$$
\sum_{j=1}^{q_{l}}\left|F\left(\left(I_{l}^{j}\right)^{\prime}\right)\right|<\varepsilon_{l} ;
$$

тогда потребуем, чтобы для всякого $l=1,2, \ldots$ разбиение $P_{l}$ являлось $\left(\delta_{l}(\mathbf{z}), 1 / 2\right)$-согласованным и связанным со множеством $B_{l}$; в частности, разбиение $P_{l}$ для всех $l$ должно удовлетворять условию (24);

V) $B_{l} \subset\left(\bigcup_{j=1}^{q_{l}} I_{l}^{j}\right)^{\circ}$ для всех $l$;

VI) любой отрезок $\Lambda$ вида $(22)$ с $a \notin\{0,1\}$, лежащий в множестве $B_{l}$ при некотором $l$, обладает тем свойством, что $\Lambda \cap\left(I_{l}^{j}\right)^{\circ}=\varnothing$ для всех $j=$ $1, \ldots, q_{l}$ (т.е. $\Lambda$ может пересекаться с $I_{l}^{j}$ только по границе последнего).

Из построения сразу вытекает

Лемма 3. Пусть последователъность разбиениц $P_{l}=\left\{\left(\mathbf{z}_{l}^{j}, I_{l}^{j}\right)\right\}_{j=1}^{q_{l}}$ удовлетворяет условиям II), V) и VI). Тогда для любой точки $\mathbf{z} \in[0,1]^{2} \backslash\left(I_{d}\right)^{2} u$ любой (z)-последовательности существует натуральное $l_{0}$, обладающее следующим свойством: для любого $l \geqslant l_{0}$ найдется $j=j(l)$ такое, что двоичный квадрат $I_{l}^{j}$ из разбиения $P_{l}$ принадлежит этой последовательности. Таким образом, существует бесконечно много квадратов $I_{l}^{j}$, принадлежсащих этой последовательности.

Продолжим доказательство теоремы. Пусть $E \subset[0,1]^{2}-$ измеримое множество положительной меры. Введем функцию

$$
T_{E}(\Delta)=\frac{\operatorname{mes}(\Delta \cap E)}{\operatorname{mes} E},
$$

определенную для любого измеримого множества $\Delta \subset[0,1]^{2}$. Очевидно, при фиксированном $E$ функция $T_{E}(\Delta)$ счетно-аддитивна на классе измеримых множеств. Если же рассматривать функцию $T_{E}(\Delta)$ лишь для $\Delta \in \mathscr{B}_{1 / 2, d}$, то $T_{E}(\Delta) \in A_{\mathscr{B}_{1 / 2, d}}$. Очевидно, для всех измеримых множеств $E$ и $\Delta$

$$
0 \leqslant T_{E}(\Delta) \leqslant 1
$$

Построим мажоранты и миноранты из определения $\left(P\left(n_{l}\right)\right)$-интеграла для функции $f(\mathbf{z})$. Пусть задано произвольное $\varepsilon>0$. Выберем натуральное $l_{1}$ настолько большим, что $\sum_{l=l_{1}}^{\infty} \varepsilon_{l}<\varepsilon / 32$. Разобьем каждый двоичный квадрат $I_{l}^{j}$ на четыре неперекрывающихся двоичных квадрата $\left\{I_{l}^{j, k}\right\}_{k=1}^{4}$ вдвое меньшего линейного размера. Пусть

$$
A_{j l}=\max _{k=1, \ldots, 4} F\left(I_{l}^{j, k}\right), \quad B_{j l}=\min _{k=1, \ldots, 4} F\left(I_{l}^{j, k}\right) .
$$

Рассмотрим функции множеств

$$
\begin{aligned}
& U(\Delta)=\sum_{l=l_{1}}^{\infty} \sum_{j=1}^{q_{l}} \sum_{k=1}^{4}\left(A_{j l}-F\left(I_{l}^{j, k}\right)\right) T_{I_{l}^{j, k}}(\Delta), \\
& V(\Delta)=\sum_{l=l_{1}}^{\infty} \sum_{j=1}^{q_{l}} \sum_{k=1}^{4}\left(F\left(I_{l}^{j, k}\right)-B_{j l}\right) T_{I_{l}^{j, k}}(\Delta) .
\end{aligned}
$$


Докажем, что функции $U$ и $V$ определены корректно, т.е. ряды в правых частях формул (26), (27) сходятся (причем абсолютно, так как все слагаемые неотрицательны). Покажем это на примере функции $U$. Из определения $A_{j l}$ и из (25) получаем

$$
0 \leqslant\left(A_{j l}-F\left(I_{l}^{j, k}\right)\right) T_{I_{l}^{j, k}}(\Delta) \leqslant A_{j l}-F\left(I_{l}^{j, k}\right)=F\left(I_{l}^{j, k_{0}(j, l)}\right)-F\left(I_{l}^{j, k}\right) .
$$

По лемме 2, примененной к функции $F$ и к двоичным квадратам $I_{l}^{j, k_{0}(j, l)}$ и $I_{l}^{j, k}$, выражение, стоящее в правой части последней формулы, не превосходит суммы модулей значений функции $F$ на не более чем восьми $1 / 2$-регулярных двоичных интервалах из основной для точки $\mathbf{z}_{l}^{j}$ последовательности. Объединив все такие двоичные интервалы для всех $k=1, \ldots, 4$ и $j=1, \ldots, q_{l}$, получим объединение не более чем $4 \cdot 8=32$ частичных разбиений квадрата $[0,1]^{2}$, связанных с множествами $B_{l}$ и являющихся $\left(\delta_{l}(\mathbf{z}), 1 / 2\right)$-согласованными. Применяя $(24)$, получаем

$$
\sum_{j=1}^{q_{l}} \sum_{k=1}^{4}\left(A_{j l}-F\left(I_{l}^{j, k}\right)\right)<32 \varepsilon_{l}
$$

откуда

$$
\sum_{l=l_{1}}^{\infty} \sum_{j=1}^{q_{l}} \sum_{k=1}^{4}\left(A_{j l}-F\left(I_{l}^{j, k}\right)\right) T_{I_{l}^{j, k}}(\Delta) \leqslant \sum_{l=l_{1}}^{\infty} 32 \varepsilon_{l}<\varepsilon .
$$

Таким образом, ряд в правой части формулы (26) сходится, и для всех измеримых множеств $\Delta$ выполнено неравенство

$$
0 \leqslant U(\Delta)<\varepsilon
$$

Аналогично, для всех измеримых множеств $\Delta$ выполнено неравенство

$$
0 \leqslant V(\Delta)<\varepsilon
$$

Заметим, что функции $U$ и $V$ конечно-аддитивны на классе измеримых множеств, в частности

$$
U(\Delta), V(\Delta) \in A_{\mathscr{B}_{1 / 2, d}} .
$$

В условиях доказательства теоремы докажем ряд утверждений.

Лемма 4. 1) Если $\Delta_{1}$ и $\Delta_{2}$ - неперекрывающиеся двоичные квадраты, то $T_{\Delta_{2}}\left(\Delta_{1}\right)=0$

2) если $\Delta_{2}$ - двоичный квадрат, то для любого набора $\left\{\Delta^{i}: i=1, \ldots, 2 k\right\}$ попарно неперекрывающихся двоччных квадратов одного ранга, лежащих в $\Delta_{2}$, выполнено равенство

$$
\sum_{i=1}^{2 k}(-1)^{i-1} T_{\Delta_{2}}\left(\Delta^{i}\right)=0 .
$$

ДокАзАтЕльство. Утверждение части 1) леммы сразу вытекает из определения функции $T$. Для доказательства части 2) получим с учетом того, что 
$\Delta^{i} \subset \Delta_{2}$ для всех $i=1, \ldots, 2 k$,

$$
\begin{gathered}
T_{\Delta_{2}}\left(\Delta^{i}\right)=\frac{\operatorname{mes}\left(\Delta^{i} \cap \Delta_{2}\right)}{\operatorname{mes} \Delta_{2}}=\frac{\operatorname{mes} \Delta^{i}}{\operatorname{mes} \Delta_{2}} \\
\sum_{i=1}^{2 k}(-1)^{i-1} T_{\Delta_{2}}\left(\Delta^{i}\right)=\frac{1}{\operatorname{mes} \Delta_{2}} \sum_{i=1}^{2 k}(-1)^{i-1} \operatorname{mes} \Delta^{i}=0,
\end{gathered}
$$

что и доказывает часть 2) леммы.

Лемма 5. Пусть $W \subset[0,1]^{2}$ - некоторое счетное множество, $\mathbf{z} \in\left(Q_{d} \times\right.$ $\left.I_{d}\right) \backslash W\left(\right.$ или $\left.\mathbf{z} \in\left(I_{d} \times Q_{d}\right) \backslash W\right), a\left\{\Delta_{n}\right\}$ - любая из $(\mathbf{z}, \square)$-последовательностей. Тогда существует такая возрастающая последовательность натуральньх чисел $n_{l}=n_{l}\left(\mathbf{z},\left\{\Delta_{n}\right\}\right)$, что для этой последовательности и для функиий $F(\Delta)+U(\Delta), F(\Delta)-V(\Delta)$ выполнено равенство (16) (или равенство (17)).

ДокАЗАТЕЛЬСтво. Будем считать, что $\mathbf{z} \in\left(I_{d} \times Q_{d}\right) \backslash W$, и рассмотрим функцию $F+U$ (остальные случаи доказываются аналогично). По лемме 3 существует последовательность двоичных квадратов $\left\{I_{l}^{j(l)}\right\}_{l \geqslant l_{1}}$ (каждый из квадратов $I_{l}^{j(l)}$ берется из разбиения $\left.P_{l}\right)$, любой из которых принадлежит $(\mathbf{z}, \square)$ последовательности $\left\{\Delta_{n}\right\}$. Положим $n_{l}=\left(\right.$ ранг квадрата $\left.I_{l}^{j(l)}\right)+1$. Тогда $I_{l}^{j(l)}=\Delta_{n_{l}-1}$. Зафиксируем $l>\max \left\{l_{0}, l_{1}\right\}$. Двоичные квадраты $\Delta^{s} \stackrel{\text { def }}{=} \Delta_{n_{l}, n_{l}}^{s}$ $(s=1,2$, нумерация квадратов соответствует условиям (4)) лежат в квадрате $I_{l}^{j(l)}$. Запишем разность, фигурирующую в формуле (17), для функции $\Phi=F+U$ :

$$
\begin{aligned}
\sum_{s=1,2}( & -1)^{s-1}\left(F\left(\Delta^{s}\right)+U\left(\Delta^{s}\right)\right) \\
= & \sum_{s=1,2}(-1)^{s-1}\left(F\left(\Delta^{s}\right)+\sum_{\alpha=l_{1}}^{\infty} \sum_{j=1}^{q_{\alpha}} \sum_{k=1}^{4}\left(A_{j \alpha}-F\left(I_{\alpha}^{j, k}\right)\right) T_{I_{\alpha}^{j, k}}\left(\Delta^{s}\right)\right) \\
= & \sum_{\alpha=l_{1}} \sum_{j=1}^{l-1} \sum_{k=1}^{q_{\alpha}}\left(A_{j \alpha}-F\left(I_{\alpha}^{j, k}\right)\right) \sum_{s=1,2}(-1)^{s-1} \frac{\operatorname{mes}\left(I_{\alpha}^{j, k} \cap \Delta^{s}\right)}{\operatorname{mes} I_{\alpha}^{j, k}} \\
& +\sum_{j=1}^{q_{l}} \sum_{k=1}^{4} \sum_{s=1,2}(-1)^{s-1}\left(F\left(\Delta^{s}\right)+\left(A_{j l}-F\left(I_{l}^{j, k}\right)\right) \frac{\operatorname{mes}\left(I_{l}^{j, k} \cap \Delta^{s}\right)}{\operatorname{mes} I_{l}^{j, k}}\right) \\
& \quad+\sum_{s=1,2}(-1)^{s-1} \sum_{\alpha=l+1}^{\infty} \sum_{j=1}^{q_{\alpha}} \sum_{k=1}^{4}\left(A_{j \alpha}-F\left(I_{\alpha}^{j, k}\right)\right) \frac{\operatorname{mes}\left(I_{\alpha}^{j, k} \cap \Delta^{s}\right)}{\operatorname{mes} I_{\alpha}^{j, k}} .
\end{aligned}
$$

Рассмотрим правую часть (32). Если для некоторых $\alpha, j, k, s$ квадраты $I_{\alpha}^{j, k}$ и $\Delta^{s}$ не перекрываются, то согласно части 1) леммы 4

$$
\frac{\operatorname{mes}\left(I_{l}^{j, k} \cap \Delta^{s}\right)}{\operatorname{mes} I_{\alpha}^{j, k}}=0 .
$$

Поэтому достаточно рассмотреть $\alpha, j, k$ и $s$ такие, что квадраты $I_{\alpha}^{j, k}$ и $\Delta^{s}$ перекрываются. 
Пусть $\alpha \leqslant l-1$ и квадрат $I_{\alpha}^{j, k}$ перекрывается с квадратом $\Delta^{s}$. Тогда по построению (см. условие II)) квадрат $I_{\alpha}^{j, k}$ покрывает квадрат $I_{l}^{j(l)}$, а значит, и оба квадрата $\Delta^{s}(s=1,2)$. Отсюда в силу части 2) леммы 4 получаем, что первое из трех слагаемых в правой части (32) равно нулю.

Пусть $\alpha=l$ и двоичные квадраты $I_{l}^{j, k}$ и $\Delta^{s}$ перекрываются. Тогда $j=j(l)$, $I_{l}^{j(l)}=\bigcup_{k=1}^{4} I_{l}^{j, k}$ и каждый из квадратов $\Delta^{s}$ совпадает с квадратом $I_{l}^{j, k(s)}$ для некоторого $k(s)$. Тогда

$$
\frac{\operatorname{mes}\left(I_{l}^{j, k(s)} \cap \Delta^{s}\right)}{\operatorname{mes} I_{l}^{j, k}}=1,
$$

откуда

$$
\begin{aligned}
\sum_{j=1}^{q_{l}} & \sum_{k=1}^{4} \sum_{s=1,2}(-1)^{s-1}\left(F\left(\Delta^{s}\right)+\left(A_{j l}-F\left(I_{l}^{j, k}\right)\right) \frac{\operatorname{mes}\left(I_{l}^{j, k} \cap \Delta^{s}\right)}{\operatorname{mes} I_{l}^{j, k}}\right) \\
& =\sum_{s=1,2}(-1)^{s-1}\left(F\left(\Delta^{s}\right)+\left(A_{j l}-F\left(I_{l}^{j, k(s)}\right)\right)\right) \\
& =\sum_{s=1,2}(-1)^{s-1}\left(F\left(\Delta^{s}\right)+\left(A_{j l}-F\left(\Delta^{s}\right)\right)\right)=\sum_{s=1,2}(-1)^{s-1} A_{j l}=0 .
\end{aligned}
$$

Таким образом, второе слагаемое в правой части (32) равно нулю.

Рассмотрим третье слагаемое в правой части (32). Так как

$$
\frac{\operatorname{mes}\left(I_{\alpha}^{j, k} \cap \Delta\right)}{\operatorname{mes} I_{\alpha}^{j, k}} \leqslant 1
$$

то, используя (28), получим, что для любого измеримого множества $\Delta$ имеет место цепочка соотношений

$$
\begin{aligned}
0 & \leqslant \sum_{\alpha=l+1}^{\infty} \sum_{j=1}^{q_{\alpha}} \sum_{k=1}^{4}\left(A_{j \alpha}-F\left(I_{\alpha}^{j, k}\right)\right) \frac{\operatorname{mes}\left(I_{\alpha}^{j, k} \cap \Delta\right)}{\operatorname{mes} I_{\alpha}^{j, k}} \\
& \leqslant \sum_{\alpha=l+1}^{\infty} \sum_{j=1}^{q_{\alpha}} \sum_{k=1}^{4}\left(A_{j \alpha}-F\left(I_{\alpha}^{j, k}\right)\right)<32 \sum_{\alpha=l+1}^{\infty} \varepsilon_{\alpha} .
\end{aligned}
$$

Применяя (33), оценим третье слагаемое в правой части (32):

$$
\left|\sum_{s=1,2}(-1)^{s-1} \sum_{\alpha=l+1}^{\infty} \sum_{j=1}^{q_{\alpha}} \sum_{k=1}^{4}\left(A_{j \alpha}-F\left(I_{\alpha}^{j, k}\right)\right) \frac{\operatorname{mes}\left(I_{\alpha}^{j, k} \cap \Delta^{s}\right)}{\operatorname{mes} I_{\alpha}^{j, k}}\right| \leqslant 64 \sum_{\alpha=l+1}^{\infty} \varepsilon_{\alpha} .
$$

Учитывая оценки трех слагаемых в правой части (32), получаем

$$
\begin{aligned}
& \left|\sum_{s=1,2}(-1)^{s-1}\left(F\left(\Delta^{s}\right)+U\left(\Delta^{s}\right)\right)\right| \leqslant 0+0+64 \sum_{\alpha=l+1}^{\infty} \varepsilon_{\alpha}
\end{aligned}
$$

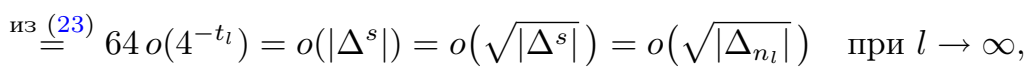

что доказывает лемму. 
Лемма 6. Функиии $U(\Delta)$ u $V(\Delta)$ являются равномерно непрерывными как функции двоччного квадрата. Это означает, что справедливы соотношения

$$
\lim _{|\Delta| \rightarrow 0} U(\Delta)=0, \quad \lim _{|\Delta| \rightarrow 0} V(\Delta)=0
$$

где $\Delta-$ двоичный квадрат.

ДокАЗАТЕЛЬСтво проведем для функции $U(\Delta)$ (для функции $V(\Delta)$ все аналогично). Выберем произвольное $\varepsilon^{\prime}>0$. Пусть натуральные $l_{2}>l_{1}$ и $n_{0}>t_{l_{2}}$ настолько велики, что

$$
\begin{array}{r}
32 \sum_{\alpha=l_{2}+1}^{\infty} \varepsilon_{\alpha}<\varepsilon^{\prime}, \\
2^{t_{l_{2}}-n_{0}} 32 \sum_{l=l_{1}}^{l_{2}} \varepsilon_{l}<\varepsilon^{\prime} .
\end{array}
$$

Возьмем любой двоичный квадрат $\Delta$ ранга большего чем $n_{0}$. Тогда

$$
\begin{aligned}
U(\Delta)= & \sum_{l=l_{1}}^{\infty} \sum_{j=1}^{q_{l}} \sum_{k=1}^{4}\left(A_{j l}-F\left(I_{l}^{j, k}\right)\right) T_{I_{l}^{j, k}}(\Delta) \\
= & \sum_{l=l_{1}}^{l_{2}} \sum_{j=1}^{q_{l}} \sum_{k=1}^{4}\left(A_{j l}-F\left(I_{l}^{j, k}\right)\right) \frac{\operatorname{mes}\left(I_{l}^{j, k} \cap \Delta\right)}{\operatorname{mes} I_{l}^{j, k}} \\
& +\sum_{l=l_{2}+1}^{\infty} \sum_{j=1}^{q_{l}} \sum_{k=1}^{4}\left(A_{j l}-F\left(I_{l}^{j, k}\right)\right) T_{I_{l}^{j, k}}(\Delta) .
\end{aligned}
$$

Оценим оба слагаемых в правой части (37). Отметим, что при фиксированном $l \leqslant l_{2}$ двоичный квадрат $\Delta$, имеющий ранг $n_{0}>t_{l_{2}}$, перекрывается не более чем с одним из квадратов $I_{l}^{j, k}, j=1, \ldots, q_{l}, k=1, \ldots, 4$. Тогда для такого квадрата $I_{l}^{j, k}$ и для квадрата $\Delta$ выполнены соотношения

$$
\frac{\operatorname{mes}\left(I_{l}^{j, k} \cap \Delta\right)}{\operatorname{mes} I_{l}^{j, k}}=\frac{\operatorname{mes} \Delta}{\operatorname{mes} I_{l}^{j, k}} \leqslant \frac{2^{-n_{0}}}{2^{-t_{l_{2}}}}=2^{t_{l_{2}}-n_{0}},
$$

откуда

$$
\begin{aligned}
& \sum_{l=l_{1}}^{l_{2}} \sum_{j=1}^{q_{l}} \sum_{k=1}^{4}\left(A_{j l}-F\left(I_{l}^{j, k}\right)\right) \frac{\operatorname{mes}\left(I_{l}^{j, k} \cap \Delta\right)}{\operatorname{mes} I_{l}^{j, k}} \leqslant 2^{t_{l_{2}}-n_{0}} \sum_{l=l_{1}}^{l_{2}} \sum_{j=1}^{q_{l}} \sum_{k=1}^{4}\left(A_{j l}-F\left(I_{l}^{j, k}\right)\right) \\
& \stackrel{\text { из (28) }}{\leqslant} 2^{t_{l_{2}}-n_{0}} 32 \sum_{l=l_{1}}^{l_{2}} \varepsilon_{l} \stackrel{\text { из }}{<} \varepsilon^{\prime},
\end{aligned}
$$

и мы получили оценку первого слагаемого правой части (37). Второе слагаемое оценивается при помощи (33) и (35):

$$
\sum_{l=l_{2}+1}^{\infty} \sum_{j=1}^{q_{l}} \sum_{k=1}^{4}\left(A_{j l}-F\left(I_{l}^{j, k}\right)\right) T_{I_{l}^{j, k}}(\Delta) \leqslant 32 \sum_{\alpha=l_{2}+1}^{\infty} \varepsilon_{\alpha}<\varepsilon^{\prime} .
$$


Объединяя $(37),(38)$ и $(39)$, получаем неравенство $0 \leqslant U(\Delta)<2 \varepsilon^{\prime}$, которое доказывает лемму.

Вернемся к доказательству теоремы 4. Из соотношений (34) и лемм 1, 2 вытекает, что функции $U(\Delta), V(\Delta)$ и $F(\Delta)$ удовлетворяют условию $(11)$. Функции $F(\Delta)+U(\Delta)$ и $F(\Delta)-V(\Delta)$ уже обладают рядом свойств мажоранты и миноранты для $\left(P\left(n_{l}\right)\right)$-интеграла: для них выполняются условия $(11),(16)$ и $(17)$. Остается добиться выполнения равенства (21). Для этого мы "исправим" данные функции, добиваясь, чтобы они продолжали удовлетворять условиям (11), (16) и (17).

Рассмотрим еще раз функцию

$$
F(\Delta)=\left(H_{1 / 2, d}\right) \int_{\Delta} f(\mathbf{z}) d \mathbf{z}
$$

По теореме А п.в. на $[0,1]^{2}$ справедливо равенство

$$
D_{\mathscr{B}_{1 / 2, d}} F(\mathbf{z})=f(\mathbf{z})
$$

Пусть $K$ - множество точек из $\left(I_{d}\right)^{2}$, для которых не выполняется равенство (40). Рассмотрим построенную в начале доказательства теоремы последовательность разбиений $P_{l}=\left\{\left(\mathbf{z}_{l}^{j}, I_{l}^{j}\right)\right\}_{j=1}^{q_{l}}$. Пусть $C_{l}$ обозначает $\bigcup_{j=1}^{q_{l}} I_{l}^{j}$. Пусть $\widetilde{K}$ обозначает множество точек $\mathbf{z} \in K$, принадлежащих $C_{l}$ для бесконечного множества значений $l$. Рассмотрим множества

$$
K_{l}=\left\{\mathbf{z} \in K: \mathbf{z} \notin \bigcup_{n \geqslant l} C_{n}\right\} .
$$

Очевидно,

$$
K_{l} \subseteq K_{l+1} \quad \text { для всех } l, \quad K=\widetilde{K} \cup\left(\bigcup_{l} K_{l}\right) .
$$

Рассмотрим выбранные в ходе доказательства теоремы $\varepsilon>0$ и последовательность $\varepsilon_{l}>0$. Пусть натуральное $l_{3}$ настолько велико, что $\sum_{l=l_{3}}^{\infty} \varepsilon_{l}<\varepsilon$. Для всех $l \geqslant l_{3}$ индукционно построим функции $\widetilde{\delta}_{l}(\mathbf{z})>0$, определенные на множестве $K$, и систему двоичных квадратов $\left\{J_{l}^{i}\right\}, l=l_{3}, l_{3}+1 \ldots, i=1,2, \ldots$, обладающие следующими свойствами.

Во-первых, пусть функция $\widetilde{\delta}_{l}(\mathbf{z})$ такова, что для любого $\left(\widetilde{\delta}_{l}(\mathbf{z}), 1 / 2\right)$-согласованного разбиения $P_{l}=\left\{\left(\mathbf{z}_{l}^{i}, J_{l}^{i}\right)\right\}_{s=1}^{N}$, связанного с множеством $K_{l}$, выполнено неравенство (24). Это возможно, так как вариационная мера $V_{F}^{1 / 2, d}$ абсолютно непрерывна и mes $K_{l}=0$ для всех $l$. Далее, при всех $l$ для каждой точки $\mathbf{z} \in K_{l}$ отметим двоичный квадрат $\Delta(l, \mathbf{z})$, для которого выполнены включения $\mathbf{z} \in \Delta(l, \mathbf{z}) \subset B\left(\mathbf{z}, \widetilde{\delta}_{l}(\mathbf{z})\right)$. Потребуем, чтобы $\Delta(l, \mathbf{z}) \cap C_{l}=\varnothing$. Это возможно, так как множество $C_{l}$ замкнуто и $K_{l} \cap C_{l}=\varnothing$. Потребуем также, чтобы при $\mathbf{z} \in K_{l-1}$ квадрат $\Delta(l, \mathbf{z})$ строго лежал в одном из квадратов вида $J_{l-1}^{i}$. Выкинув из набора $\{\Delta(l, \mathbf{z})\}$ двоичные квадраты, лежащие в других двоичных квадратах из этого набора, получим для заданного $l$ набор $\left\{J_{l}^{i}\right\}$. 
Отметим, что для любого $l \geqslant l_{3}$ квадраты $J_{l}^{i}, i=1,2, \ldots$, попарно не перекрываются и их объединение покрывает множество $K_{l}$. Далее, для любого $l \geqslant l_{3}$ и любого натурального $N$ система квадратов $\left\{J_{l}^{i}\right\}_{i=1}^{N}$ образует $\left(\widetilde{\delta}_{l}(\mathbf{z}), 1 / 2\right)$-согласованное разбиение, связанное с множеством $K_{l}$. Это означает, в частности, что выполнено неравенство

$$
\sum_{i=1}^{N}\left|F\left(J_{l}^{i}\right)\right|<\varepsilon_{l}, \quad \text { откуда } \sum_{i}\left|F\left(J_{l}^{i}\right)\right| \leqslant \varepsilon_{l} .
$$

Кроме того, для любого $l \geqslant l_{3}$ множество $\bigcup_{i} J_{l}^{i}$ не пересекается с множеством $\bigcup_{j=1}^{q_{l}} I_{l}^{j}$, построенным в начале теоремы. Далее,

$(\star)$ любая точка $\mathbf{z} \in K_{l_{0}}$ покрыта бесконечным числом квадратов $J_{l}^{i(l)}, l \geqslant l_{0}$.

Построим функцию множества $\gamma_{1}(\Delta)$ по формуле

$$
\gamma_{1}(\Delta)=\sum_{l=l_{3}}^{\infty} \sum_{j=1}^{q_{l}}\left|F\left(I_{l}^{j}\right)\right| T_{I_{l}^{j}}(\Delta)+\sum_{l=l_{3}}^{\infty} \sum_{i}\left|F\left(J_{l}^{i}\right)\right| T_{J_{l}^{i}}(\Delta),
$$

где, как и ранее, $T_{E}(\Delta)=\operatorname{mes}(E \cap \Delta) / \operatorname{mes} E$. Отметим, что ряды в правой части (41) знакоположительные и

$$
\begin{aligned}
& \sum_{l=l_{3}}^{\infty} \sum_{j=1}^{q_{l}}\left|F\left(I_{l}^{j}\right)\right| T_{J_{l}^{i}}(\Delta)+\sum_{l=l_{3}}^{\infty} \sum_{i}\left|F\left(J_{l}^{i}\right)\right| T_{J_{l}^{i}}(\Delta) \\
& \quad \leqslant \sum_{l=l_{3}}^{\infty} \sum_{j=1}^{q_{l}}\left|F\left(I_{l}^{j}\right)\right|+\sum_{l=l_{3}}^{\infty} \sum_{i}\left|F\left(J_{l}^{i}\right)\right| \leqslant 2 \sum_{l=l_{3}}^{\infty} \varepsilon_{l}<2 \varepsilon
\end{aligned}
$$

т.е. эти ряды сходятся, функция $\gamma_{1}$ определена корректно и для любого измеримого множества $\Delta$ выполнено условие

$$
0 \leqslant \gamma_{1}(\Delta)<2 \varepsilon
$$

Отметим также, что функция $\gamma_{1}$ конечно-аддитивна на классе измеримых множеств, в частности,

$$
\gamma_{1}(\Delta) \in A_{\mathscr{B}_{1 / 2, d}}
$$

В рамках доказательства теоремы докажем следующие леммы.

Лемма 7. Пусть $\mathbf{z} \in K, a\left\{\Delta_{n}\right\}-(\mathbf{z}, \square)$-последовательность. Тогда существует возрастающая последовательность натуральных чисел $n_{l}$ такая, что для функиий $F(\Delta) \pm \gamma_{1}(\Delta)$ выполнены неравенства

$$
\varliminf_{l \rightarrow \infty} \frac{\left(F+\gamma_{1}\right)\left(\Delta_{n_{l}}\right)}{\left|\Delta_{n_{l}}\right|} \geqslant 0 \geqslant \varlimsup_{l \rightarrow \infty} \frac{\left(F-\gamma_{1}\right)\left(\Delta_{n_{l}}\right)}{\left|\Delta_{n_{l}}\right|} .
$$

ДокАзАТЕльство. Докажем левое неравенство в формуле (44) (правое доказывается аналогично). Зафиксируем точку $\mathbf{z} \in K$. Возможны два случая. 
Пусть $\mathbf{z} \in \widetilde{K}$. Тогда для всех $l>\max \left\{l_{1}, l_{3}\right\} \mathbf{z} \in I_{l}^{j(\mathbf{z}, l)}$ при некотором $j(\mathbf{z}, l)$. Положим $\Delta_{n_{l}}=I_{l}^{j(\mathbf{z}, l)}$. Зафиксируем произвольное $l>l_{3}$. Имеем

$$
\begin{aligned}
\left(F+\gamma_{1}\right)\left(\Delta_{n_{l}}\right) & =\left(F\left(I_{l}^{j(\mathbf{z}, l)}\right)+\left|F\left(I_{l}^{j(\mathbf{z}, l)}\right)\right| T_{I_{l}^{j(\mathbf{z}, l)}}\left(I_{l}^{j(\mathbf{z}, l)}\right)\right) \\
& +\sum_{I_{\alpha}^{j} \neq I_{l}^{j(\mathbf{z}, l)}}\left|F\left(I_{\alpha}^{j}\right)\right| T_{I_{\alpha}^{j}}\left(I_{l}^{j(\mathbf{z}, l)}\right)+\sum_{\alpha=l_{3}}^{\infty} \sum_{i}\left|F\left(J_{\alpha}^{i}\right)\right| T_{J_{\alpha}^{i}}\left(J_{l}^{i(\mathbf{z}, l)}\right) .
\end{aligned}
$$

Рассмотрим правую часть (45). Так как по определению функции $T_{E}(\Delta)$ справедливо равенство

$$
T_{I_{l}^{j(\mathbf{z}, l)}}\left(I_{l}^{j(\mathbf{z}, l)}\right)=1
$$

то

$$
F\left(I_{l}^{j(\mathbf{z}, l)}\right)+\left|F\left(I_{l}^{j(\mathbf{z}, l)}\right)\right| T_{I_{l}^{j(\mathbf{z}, l)}}\left(I_{l}^{j(\mathbf{z}, l)}\right)=F\left(I_{l}^{j(\mathbf{z}, l)}\right)+\left|F\left(I_{l}^{j(\mathbf{z}, l)}\right)\right| \geqslant 0 .
$$

Из определения функции $T_{E}(\Delta)$ вытекает, что второе и третье слагаемые также неотрицательны. Следовательно, выполнено неравенство $\left(F+\gamma_{1}\right)\left(\Delta_{n_{l}}\right) \geqslant 0$, и для таких точек $\mathbf{z}$ получаем утверждение леммы.

Во втором случае $\mathbf{z} \in K_{l_{4}}$ для некоторого натурального $l_{4}$. Тогда согласно $(\star)$ для всех $l>\max \left\{l_{1}, l_{3}, l_{4}\right\} \mathbf{z} \in J_{l}^{i(\mathbf{z}, l)}$ при некотором $i(\mathbf{z}, l)$. Положим $\Delta_{n_{l}}=J_{l}^{i(\mathbf{z}, l)}$. Тогда

$$
\begin{aligned}
\left(F+\gamma_{1}\right)\left(\Delta_{n_{l}}\right) & =\left(F\left(J_{l}^{i(\mathbf{z}, l)}\right)+\left|F\left(J_{l}^{i(\mathbf{z}, l)}\right)\right| T_{J_{l}^{i(\mathbf{z}, l)}}\left(J_{l}^{i(\mathbf{z}, l)}\right)\right) \\
& +\sum_{J_{\alpha}^{i} \neq J_{l}^{i(\mathbf{z}, l)}}\left|F\left(J_{\alpha}^{i}\right)\right| T_{J_{\alpha}^{i}}\left(J_{l}^{i(\mathbf{z}, l)}\right)+\sum_{\alpha=l_{1}}^{\infty} \sum_{j=1}^{q_{l}}\left|F\left(I_{\alpha}^{j}\right)\right| T_{I_{\alpha}^{j}}\left(I_{l}^{j(\mathbf{z}, l)}\right) .
\end{aligned}
$$

Рассмотрим правую часть (46). Так как $T_{J_{l}^{i(\mathbf{z}, l)}}\left(J_{l}^{i(\mathbf{z}, l)}\right)=1$, то

$$
F\left(J_{l}^{i(\mathbf{z}, l)}\right)+\left|F\left(J_{l}^{i(\mathbf{z}, l)}\right)\right| T_{J_{l}^{i(\mathbf{z}, l)}}\left(J_{l}^{i(\mathbf{z}, l)}\right)=F\left(J_{l}^{i(\mathbf{z}, l)}\right)+\left|F\left(J_{l}^{i(\mathbf{z}, l)}\right)\right| \geqslant 0,
$$

т.е. первое слагаемое в правой части (46) неотрицательно. Из определения функции $T_{E}(\Delta)$ вытекает, что второе и третье слагаемые также неотрицательны. Следовательно, $\left(F+\gamma_{1}\right)\left(\Delta_{n_{l}}\right) \geqslant 0$, и для таких точек $\mathbf{z}$ получаем утверждение леммы. Лемма доказана.

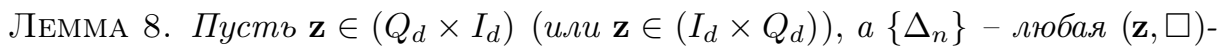
последовательность. Тогда для последовательности $n_{l}$, выбранной в лемме 5 , для последовательности $\left\{\Delta_{n}\right\}$ и функиии $\gamma_{1}$ выполняется равенство (16) (или равенство (17)).

ДокАЗАтЕЛЬство. Будем считать, что $\mathbf{z} \in\left(I_{d} \times Q_{d}\right) \backslash W$ (второй случай доказывается аналогично). Зафиксируем точку $\mathbf{z} \in\left(I_{d} \times Q_{d}\right)$ и $(\mathbf{z}, \square)$-последовательность $\left\{\Delta_{n}\right\}$. По лемме 3 существует последовательность двоичных квадратов $\left\{I_{l}^{j(l)}\right\}_{l \geqslant l_{1}}$ (каждый из квадратов $I_{l}^{j(l)}$ берется из разбиения $P_{l}$ ), любой из которых принадлежит последовательности $\left\{\Delta_{n}\right\}$. Положим, как и в лемме $5, n_{l}=\left(\right.$ ранг квадрата $\left.I_{l}^{j(l)}\right)+1$. Тогда $I_{l}^{j(l)}=\Delta_{n_{l}-1}$. Двоичные квадраты 
$\Delta^{s} \stackrel{\text { def }}{=} \Delta_{n_{l}}^{s}, s=1,2$, лежат в квадрате $I_{l}^{j(l)}$. Рассмотрим выражение под знаком суммы в формуле $(16)$ для функции $\gamma_{1}(\Delta)$. Оно имеет вид

$$
\begin{aligned}
& \sum_{s=1,2}(-1)^{s-1} \gamma_{1}\left(\Delta^{s}\right) \\
& =\sum_{s=1,2}(-1)^{s-1} \sum_{\alpha=l_{3}}^{\infty} \sum_{j=1}^{q_{\alpha}}\left|F\left(I_{\alpha}^{j}\right)\right| T_{I_{\alpha}^{j}}\left(\Delta^{s}\right)+\sum_{s=1,2}(-1)^{s-1} \sum_{\alpha=l_{3}}^{\infty} \sum_{i}\left|F\left(J_{\alpha}^{i}\right)\right| T_{J_{\alpha}^{i}}\left(\Delta^{s}\right) \\
& =\sum_{\alpha=l_{3}} \sum_{j=1}^{q_{\alpha}}\left|F\left(I_{\alpha}^{j}\right)\right| \sum_{s=1,2}(-1)^{s-1} T_{I_{\alpha}^{j}}\left(\Delta^{s}\right)+\sum_{s=1,2}(-1)^{s-1} \sum_{\alpha=l+1}^{\infty} \sum_{j=1}^{q_{\alpha}}\left|F\left(I_{\alpha}^{j}\right)\right| T_{I_{\alpha}^{j}}\left(\Delta^{s}\right) \\
& \quad+\sum_{\alpha=l_{3}}^{\infty} \sum_{i}\left|F\left(J_{\alpha}^{i}\right)\right| \sum_{s=1,2}(-1)^{s-1} T_{J_{\alpha}^{i}}\left(\Delta^{s}\right) .
\end{aligned}
$$

Оценим три слагаемых в правой части (47). При $\alpha \leqslant l$ и любом $j=1, \ldots, q_{\alpha}$ имеем $\bigcup_{s=1,2} \Delta^{s} \subset I_{\alpha}^{j}$. Согласно части 2) леммы 4

$$
\sum_{s=1,2}(-1)^{s-1} T_{I_{\alpha}^{j}}\left(\Delta^{s}\right)=0,
$$

откуда первое слагаемое в правой части (47) равно нулю. Дословно повторяя рассуждения леммы 5 , можно показать, что второе слагаемое есть $o\left(1 / 4^{t_{l}}\right)=$ $o\left(\left|\Delta^{s}\right|\right)$. Для оценки третьего слагаемого в правой части (47) заметим, что любой двоичный квадрат $J_{\alpha}^{i}$ не лежит ни в одном двоичном квадрате $\Delta^{s}$, так как по построению $J_{\alpha}^{i} \cap C_{l}=\varnothing$, a $\Delta^{s} \subset C_{l}$. Тогда либо эти двоичные квадраты не перекрываются, либо $\bigcup_{s=1,2} \Delta^{s} \subseteq J_{\alpha}^{i}$. Как показано в лемме 4 , в обоих случаях

$$
\sum_{s=1,2}(-1)^{s-1} T_{J_{\alpha}^{i}}\left(\Delta^{s}\right)=0
$$

откуда следует, что третье слагаемое в правой части (47) равно нулю. Таким образом, правая, а значит, и левая часть $(47)$ есть $o\left(\left|\Delta^{s}\right|\right)=o\left(\sqrt{\left|\Delta^{s}\right|}\right)$, что доказывает лемму.

ЛЕмма 9. Функиия $\gamma_{1}(\Delta)$ является равномерно непрерывной как функиия двоичного квадрата, т.е. имеет место равенство

$$
\lim _{|\Delta| \rightarrow 0} \gamma_{1}(\Delta)=0, \quad \text { где } \Delta-\text { двоичный квадрат. }
$$

ДокАЗАтЕЛЬСтво. Рассмотрим функцию $\gamma_{1}$, определенную формулой (41). Дословно повторяя рассуждения из леммы 6 , можно доказать, что первое слагаемое в правой части (41) равномерно непрерывно. Для оценки второго слагаемого в (41) выберем произвольное $\varepsilon^{\prime}>0$. Пусть натуральное $l_{5}>l_{3}$ настолько велико, что

$$
\sum_{\alpha=l_{5}+1}^{\infty} \varepsilon_{\alpha}<\varepsilon^{\prime}
$$


Выберем натуральное $i_{0}$ настолько большим, что для всех $l=l_{3}, \ldots, l_{5}$ имеет место неравенство

$$
\sum_{i>i_{0}}\left|F\left(J_{l}^{i}\right)\right|<\frac{\varepsilon^{\prime}}{l_{5}-l_{3}+1} .
$$

Возьмем натуральное $n_{0}$ таким, что

$$
2^{t-n_{0}} \sum_{l=l_{3}}^{l_{5}} \varepsilon_{l}<\varepsilon^{\prime}
$$

где $t$ - максимальный из рангов квадратов $J_{l}^{i}$ при $l=l_{3}, \ldots, l_{5}$ и $i \leqslant i_{0}$.

Рассмотрим произвольный двоичный квадрат $\Delta$ ранга большего, чем $n_{0}$. Тогда

$$
\begin{aligned}
\sum_{l=l_{3}}^{\infty} \sum_{i} \mid & F\left(J_{l}^{i}\right)\left|T_{J_{l}^{i}}(\Delta)=\sum_{l=l_{3}}^{l_{5}} \sum_{i \leqslant i_{0}}\right| F\left(J_{l}^{i}\right) \mid T_{J_{l}^{i}}(\Delta) \\
& +\sum_{l=l_{3}}^{l_{5}} \sum_{i>i_{0}}\left|F\left(J_{l}^{i}\right)\right| T_{J_{l}^{i}}(\Delta)+\sum_{l=l_{5}+1}^{\infty} \sum_{i}\left|F\left(J_{l}^{i}\right)\right| T_{J_{l}^{i}}(\Delta) .
\end{aligned}
$$

Оценим все три слагаемых в правой части (52). Отметим, что при $l \leqslant l_{5}$ и фиксированном $i$ двоичный квадрат $\Delta$ перекрывается не более чем с одним из квадратов $J_{l}^{i}$. Тогда для такого квадрата $J_{l}^{i}$ и квадрата $\Delta$ выполнено соотношение

$$
T_{J_{l}^{i}}(\Delta)=\frac{\operatorname{mes}\left(J_{l}^{i} \cap \Delta\right)}{\operatorname{mes} J_{l}^{i}}=\frac{\operatorname{mes} \Delta}{\operatorname{mes} J_{l}^{i}} \leqslant \frac{2^{-n_{0}}}{2^{-t}}=2^{t-n_{0}},
$$

откуда

$$
\begin{aligned}
& 0 \leqslant \sum_{l=l_{3}}^{l_{5}} \sum_{i \leqslant i_{0}}\left|F\left(J_{l}^{i}\right)\right| T_{J_{l}^{i}}(\Delta) \leqslant 2^{t-n_{0}} \sum_{l=l_{3}}^{l_{5}} \sum_{i \leqslant i_{0}}\left|F\left(J_{l}^{i}\right)\right| \\
& \leqslant 2^{t-n_{0}} \sum_{l=l_{3}}^{l_{5}} \varepsilon_{l} \stackrel{\text { по }}{<}{ }^{\prime} \text {. }
\end{aligned}
$$

Второе слагаемое из (52) оценивается так:

$$
0 \leqslant \sum_{l=l_{3}}^{l_{5}} \sum_{i \geqslant i_{0}}\left|F\left(J_{l}^{i}\right)\right| T_{J_{l}^{i}}(\Delta) \leqslant \sum_{l=l_{3}}^{l_{5}} \sum_{i \geqslant i_{0}}\left|F\left(J_{l}^{i}\right)\right| \stackrel{\text { по (50) }}{<} \varepsilon^{\prime} .
$$

Оценим третье слагаемое в (52):

$$
0 \leqslant \sum_{l=l_{5}+1}^{\infty} \sum_{i}\left|F\left(J_{l}^{i}\right)\right| T_{J_{l}^{i}}(\Delta) \leqslant \sum_{l=l_{5}+1}^{\infty} \sum_{i}\left|F\left(J_{l}^{i}\right)\right|<\sum_{l=l_{5}+1}^{\infty} \varepsilon_{l} \stackrel{\text { по }}{<} \varepsilon^{\prime} .
$$

Объединяя выражения (52)-(55), получаем

$$
0 \leqslant \sum_{l=l_{3}}^{\infty} \sum_{i}\left|F\left(J_{l}^{i}\right)\right| T_{J_{l}^{i}}(\Delta)<3 \varepsilon^{\prime}
$$

что доказывает лемму. 
Следующая лемма обобщает известный результат о производных функций (см. [20; гл. 8, §2]).

Лемма 10. Пусть $E \subset[0,1]^{2}$ - множество нулевой меръ. Тогда для произвольного $\varepsilon^{\prime \prime}>0$ существует функиия множества $\gamma_{2}(\Delta) \in A_{\mathscr{B}_{1 / 2, d}}$, для которой выполнены следующие условия:

1) $0 \leqslant \gamma_{2}(\Delta)<\varepsilon^{\prime \prime}$ для любого измеримого множества $\Delta$;

2) $D_{\mathscr{B}_{1, d}} \gamma_{2}(\mathbf{z})=+\infty$ для любой точки $\mathbf{z} \in E$;

3) для любого $\beta>0 \gamma_{2}(\Delta)=o\left(|\Delta|^{1-\beta}\right)$ nри $|\Delta| \rightarrow 0, \Delta-$ двоичный квадрат.

ДокАЗАтЕльство. Построим открытые множества $G_{k}, k=1,2, \ldots$, такие, что $G_{k} \supset E$ и mes $G_{k}<\varepsilon^{\prime \prime} / 2^{k}$. Положим $\gamma_{2}(\Delta)=\sum_{k=1}^{\infty} \operatorname{mes}\left(\Delta \cap G_{k}\right)$. Очевидно, что для функции $\gamma_{2}$ выполнено условие 1$)$. Кроме того, $\gamma_{2} \in A_{\mathscr{B}_{1 / 2, d}}$.

Зафиксируем точку $\mathbf{z} \in E$. Возьмем любую $(\mathbf{z}, \square)$-последовательность $\left\{\Delta_{n}\right\}$ и произвольное натуральное $K$. Тогда для достаточно больших $n$ выполнены включения $\Delta_{n} \subset G_{k}$ при $k=1, \ldots, K$. Отсюда $\gamma_{2}\left(\Delta_{n}\right) \geqslant K\left|\Delta_{n}\right|$, следовательно, $\gamma_{2}\left(\Delta_{n}\right) /\left|\Delta_{n}\right| \geqslant K$. Значит, $\underline{D}_{\mathscr{B}_{1, d}} \gamma_{2}(\mathbf{z}) \geqslant K$. В силу произвольности $K$ справедливо равенство $D_{\mathscr{B}_{s, 1, d}} \gamma_{2}(\mathbf{z})=+\infty$, т.е. выполнено условие 2$)$.

Возьмем произвольные $\beta>0$ и двоичный квадрат $\Delta_{n}$ ранга $n$. Тогда

$$
\begin{aligned}
\gamma_{2}\left(\Delta_{n}\right) & =\sum_{k=1}^{2 n} \operatorname{mes}\left(\Delta_{n} \cap G_{k}\right)+\sum_{k=2 n+1}^{\infty} \operatorname{mes}\left(\Delta_{n} \cap G_{k}\right) \\
& \leqslant 2 n \operatorname{mes} \Delta_{n}+\sum_{k=2 n+1}^{\infty} \operatorname{mes} G_{k} \\
& <\frac{2 n}{4^{n}}+\sum_{k=2 n+1}^{\infty} \frac{\varepsilon^{\prime \prime}}{2^{k}}=\frac{2 n+\varepsilon^{\prime \prime}}{4^{n}}=o\left(\left|\Delta_{n}\right|^{1-\beta}\right) \quad \text { при } n \rightarrow \infty,
\end{aligned}
$$

откуда следует условие 3). Лемма доказана.

Продолжим доказательство теоремы 4. Применив лемму 10, построим функцию $\gamma_{2}(\Delta)$, взяв в качестве множества $E$ множество точек, для которых не выполняется условие $(40)$, а в качестве $\varepsilon^{\prime \prime}-$ уже выбранное $\varepsilon>0$. Рассмотрим функции $F_{1}(\Delta)=\left(F+U+\gamma_{1}+\gamma_{2}\right)(\Delta)$ и $F_{2}(\Delta)=\left(F-V-\gamma_{1}-\gamma_{2}\right)(\Delta)$. Покажем, что эти функции есть соответственно мажоранта и миноранта из определения $\left(P\left(n_{l}\right)\right)$-интеграла.

В силу аддитивности функций $F, U, V, \gamma_{1}$ и $\gamma_{2}$ (см., в частности, (31) и (43)) $F_{1}, F_{2} \in A_{\mathscr{B}_{1 / 2, d}}$. Далее, пусть $\mathbf{z} \in\left(I_{d}\right)^{2}$ и $\left\{\Delta_{n}\right\}-(\mathbf{z}, \square)$-последовательность. Пусть для точки $\mathbf{z}$ выполнено равенство (40). Тогда, используя неотрицательность функций $U, \gamma_{1}$ и $\gamma_{2}$, для любой последовательности $n_{l}$ получим

$$
\begin{aligned}
\varliminf_{l \rightarrow \infty} \frac{F_{1}\left(\Delta_{n_{l}}\right)}{\left|\Delta_{n_{l}}\right|} & \geqslant \varliminf_{l \rightarrow \infty} \frac{F\left(\Delta_{n_{l}}\right)}{\left|\Delta_{n_{l}}\right|}+\varliminf_{l \rightarrow \infty} \frac{U\left(\Delta_{n_{l}}\right)}{\left|\Delta_{n_{l}}\right|}+\varliminf_{l \rightarrow \infty} \frac{\gamma_{1}\left(\Delta_{n_{l}}\right)}{\left|\Delta_{n_{l}}\right|}+\varliminf_{l \rightarrow \infty} \frac{\gamma_{2}\left(\Delta_{n_{l}}\right)}{\left|\Delta_{n_{l}}\right|} \\
& \geqslant f(\mathbf{z})+0+0+0=f(\mathbf{z}) .
\end{aligned}
$$

Если же для точки $\mathbf{z}$ не выполняется равенство (40), то, используя неравенства (44), условие 2) леммы 10 и неотрицательность функции $U(\Delta)$, получим, 
что для выбранной в ходе доказательства теоремы последовательности $n_{l}$

$$
\begin{aligned}
\varliminf_{l \rightarrow \infty} \frac{F_{1}\left(\Delta_{n_{l}}\right)}{\left|\Delta_{n_{l}}\right|} & \geqslant \varliminf_{l \rightarrow \infty} \frac{(F+U)\left(\Delta_{n_{l}}\right)}{\left|\Delta_{n_{l}}\right|}+\varliminf_{l \rightarrow \infty} \frac{\gamma_{1}\left(\Delta_{n_{l}}\right)}{\left|\Delta_{n_{l}}\right|}+\varliminf_{l \rightarrow \infty} \frac{\gamma_{2}\left(\Delta_{n_{l}}\right)}{\left|\Delta_{n_{l}}\right|} \\
& \geqslant 0+0+\infty=+\infty \geqslant f(\mathbf{z}) .
\end{aligned}
$$

Из (56) и (57) вытекает, что для всех $\mathbf{z} \in\left(I_{d}\right)^{2}$ существует последовательность $n_{l}=n_{l}(\mathbf{z})$, для которой выполнено неравенство

$$
\varliminf_{l \rightarrow \infty} \frac{F_{1}\left(\Delta_{n_{l}}\right)}{\left|\Delta_{n_{l}}\right|} \geqslant f(\mathbf{z}) .
$$

Аналогично, для всех $\mathbf{z} \in\left(I_{d}\right)^{2}$ и той же последовательности $n_{l}$ выполнено неравенство

$$
f(\mathbf{z}) \geqslant \varliminf_{l \rightarrow \infty} \frac{F_{2}\left(\Delta_{n_{l}}\right)}{\left|\Delta_{n_{l}}\right|} .
$$

Формулы (58), (59) означают выполнение свойства 1$)$ из определения $\left(P\left(n_{l}\right)\right)$ интеграла.

В силу лемм 5 и 9 функции $F+U, F-V$ и $\gamma_{1}$ удовлетворяют условиям (16) и (17) для некоторого допустимого набора $n_{l}$. В силу леммы 10 функция $\gamma_{2}$ удовлетворяет этим же условиям для любого набора $n_{l}$. Следовательно, формулы (16) и (17), т.е. свойство 2) из определения $\left(P\left(n_{l}\right)\right)$-интеграла, выполнены и для функций $F_{1}$ и $F_{2}$.

Из утверждений лемм 1, 2 и 10, а также из (34), (48) следует, что для функций $F, U, V, \gamma_{1}$ и $\gamma_{2}$ справедливо равенство (11). Значит, оно имеет место и для функций $F_{1}$ и $F_{2}$. Таким образом, свойство 3$)$ из определения $\left(P\left(n_{l}\right)\right)$-интеграла выполнено. Наконец, в силу (29), (30), (42) и (56) для любого двоичного интервала $\Delta$ имеют место неравенства

$$
F_{1}(\Delta)-F_{2}(\Delta)=U(\Delta)+V(\Delta)+2 \gamma_{1}(\Delta)+2 \gamma_{2}(\Delta) \leqslant 2 \varepsilon+2 \varepsilon+6 \varepsilon+2 \varepsilon=12 \varepsilon,
$$

откуда следует выполнение свойства 4$)$ из определения $\left(P\left(n_{l}\right)\right)$-интеграла. Значит, функция $f(\mathbf{z})$ является $\left(P\left(n_{l}\right)\right)$-интегрируемой. Заметив, что

$$
\begin{aligned}
\left(P\left(n_{l}\right)\right) \int_{\Delta} f(\mathbf{z}) d \mathbf{z} & =\inf _{F_{1}} F_{1}(\Delta) \leqslant \inf _{U, \gamma_{1}, \gamma_{2}}\left(F+U+\gamma_{1}+\gamma_{2}\right)(\Delta) \\
& =F(\Delta)=\left(H_{1 / 2, d}\right) \int_{\Delta} f(\mathbf{z}) d \mathbf{z}=F(\Delta) \\
& =\sup _{V, \gamma_{1}, \gamma_{2}}\left(F-V-\gamma_{1}-\gamma_{2}\right)(\Delta) \leqslant \sup _{F_{2}} F_{2}(\Delta) \\
& =\left(P\left(n_{l}\right)\right) \int_{\Delta} f(\mathbf{z}) d \mathbf{z},
\end{aligned}
$$

получим, что $\left(P\left(n_{l}\right)\right)$-интеграл и $\left(H_{1 / 2, d}\right)$-интеграл от функции $f(\mathbf{z})$ совпадают. Теорема доказана.

Отметим, что для $0<\rho_{1}<\rho_{2} \leqslant 1$ выполнено включение $P_{\rho_{1}} \subset P_{\rho_{2}}$, причем оно строгое (см. [16]). Из этого факта, из включения (3) и теоремы А следует, что при $\rho \in(0,1 / 2]$ справедливы включения

$$
P_{\rho} \subset P_{1 / 2} \subset P_{1 / 2, d}=H_{1 / 2, d} .
$$


Теорема 5. Пусть $\rho \in(0,1 / 2]$. Тогда непротиворечивыми будут являться $\left(H_{1 / 2, d}\right)$-интеграл и $\left(P_{d}^{\rho, *}\right)$-интеграл, а также $\rho$-регулярный интеграл Перрона и $\left(P_{d}^{\rho, *}\right)$-интеграл.

ДокАЗАТЕЛЬство. Пусть функция $f(\mathbf{z})$ является одновременно $\left(P_{d}^{\rho, *}\right)-$ и $\left(H_{1 / 2, d}\right)$-интегрируемой. По теореме $4\left(H_{1 / 2, d}\right)$-интеграл от функции $f(\mathbf{z})$ совпадает с $\left(P\left(n_{l}\right)\right)$-интегралом от этой же функции для некоторого допустимого набора $n_{l}$. С другой стороны, по теореме 3 при любом допустимом наборе $n_{l}\left(P_{d}^{\rho, *}\right)$-интеграл и $\left(P\left(n_{l}\right)\right)$-интеграл от функции $f(\mathbf{z})$ совпадают. Тогда $\left(P_{d}^{\rho, *}\right)$-интеграл и $\left(H_{1 / 2, d}\right)$-интеграл от данной функции $f(\mathbf{z})$ совпадают. Непротиворечивость $\left(H_{1 / 2, d}\right)$ - и $\left(P_{d}^{\rho, *}\right)$-интегралов доказана. Из этого факта и из $(60)$ вытекает непротиворечивость $\rho$-регулярного интеграла Перрона и $\left(P_{d}^{\rho, *}\right)$-интеграла.

\section{§ 6. Теоремы типа Дю Буа-Реймона для двойных рядов Хаара}

Здесь представлены результаты, относящиеся к теории единственности двойных рядов Хаара.

Теорема 6. Пусть заданы $\rho \in(0,1 / 2]$ и не более чем счетное множество $W \subset[0,1]^{2}$. Предположим, что ряд (1) всюду на $[0,1]^{2}$ удовлетворяет одному из условий (12) или (13). Пусть во всех точках множества $\left(I_{d}\right)^{2} \backslash W$ данный ряд сходится $\rho$-регулярно или по прямоугольникам к конечной функции $f(\mathbf{z})$, интегрируемой либо по Перрону в $\rho$-регулярном смысле, либо в смысле $\left(H_{1 / 2, d}\right)$-интеграла, а во всех точках $\mathbf{z} \in[0,1]^{2} \backslash W$, имеющих ровно одну $Q_{d}$-координату, для данного ряда выполняется условие (14). Тогда ряд (1) является рядом Фуръе-Перрона (Фуръе-Хенстока) функиии $f(\mathbf{z})$.

ДокАзАтЕльство. Пусть выполнены условия теоремы. По теореме С функция $f(\mathbf{z})$ является $\left(P_{d}^{\rho, *}\right)$-интегрируемой и для всех $n, m$ верно равенство

$$
a_{n, m}=\left(P_{d}^{\rho, *}\right) \int_{[0,1]^{2}} f(\mathbf{z}) \chi_{n, m}(\mathbf{z}) d \mathbf{z} .
$$

Пусть функция $f(\mathbf{z})$, а значит, и функция $f(\mathbf{z}) \chi_{n, m}(\mathbf{z})$ являются также и $\left(H_{1 / 2, d}\right)$-интегрируемыми. По теореме 5

$$
\left(P_{d}^{\rho, *}\right) \int_{[0,1]^{2}} f(\mathbf{z}) \chi_{n, m}(\mathbf{z}) d \mathbf{z}=\left(H_{1 / 2, d}\right) \int_{[0,1]^{2}} f(\mathbf{z}) \chi_{n, m}(\mathbf{z}) d \mathbf{z} .
$$

Из равенств (61), (62) получаем

$$
a_{n, m}=\left(H_{1 / 2, d}\right) \int_{[0,1]^{2}} f(\mathbf{z}) \chi_{n, m}(\mathbf{z}) d \mathbf{z},
$$

откуда для функций $f \in H_{1 / 2, d}$ следует утверждение теоремы. Для функций $f \in P_{\rho}$ утверждение теоремы следует из (60) и (63). 


\section{Из теоремы 6 вытекает}

Tеорема 7. Пусть при $\rho \in(0,1 / 2]$ ряд (1) всюду на $[0,1]^{2}$ сходится по прямоугольникам или $\rho$-регулярно к конечной функиии $f(\mathbf{z})$, интегрируемой либо по Перрону в $\rho$-регулярном смысле, либо в смысле $\left(H_{1 / 2, d}\right)$-интеграла. Тогда данный ряд есть ряд Фуръе-Перрона (Фуръе-Хенстока) функиии $f(\mathbf{z})$.

\section{Список литературы}

[1] P. du Bois-Reymond, "Beweis, dass die Coefficienten der trigonometrischen Reihe $f(x)=\Sigma\left(a_{p} \cos p x+b_{p} \sin . p x\right)$ die Werthe $a_{0}=\frac{1}{2 \pi} \int_{\pi}^{+\pi} d \alpha f(\alpha), a_{p}=$ $\frac{1}{\pi} \int_{\pi}^{+\pi} d \alpha f(\alpha) \cos . p \alpha, b_{p}=\frac{1}{\pi} \int_{\pi}^{+\pi} d \alpha f(\alpha) \sin . p \alpha$, haben, jedesmal, wenn diese Integrale endlich und bestimmt sind", Munch. Abh., XII:1 (1876), 117-166.

[2] Н.К. Бари, Тригонометрические ряды, Физматгиз, М., 1961; англ. пер.: N. K. Bary, A treatise on trigonometric series, vols. I, II, Pergamon Press, OxfordLondon-New York-Paris-Frankfurt, 1964.

[3] В. А. Скворцов, "О множествах единственности для многомерных рядов Хаара", Матем. заметки, 14:6 (1973), 789-798; англ. пер.: V. A. Skvortsov, "Uniqueness sets for multiple Haar series", Math. Notes, 14:6 (1973), 1011-1016.

[4] В. А. Скворцов, "Об одной теореме единственности для многомерного ряда Хаара", Изв. АН АрмССР. Матем., 23:3 (1988), 293-296; англ. пер.: V. A. Skvortsov, "On a uniqueness theorem for a multidimensional Haar series", Soviet J. Contemporary Math. Anal., 23:3 (1988), 104-108.

[5] В. А. Скворцов, А. А. Талалян, "Некоторые вопросы единственности кратных рядов по системе Хаара и тригонометрической системе", Матем. заметки, 46:2 (1989), 104-113; англ. пер.: V.A. Skvortsov, A. A. Talalyan, "Some uniqueness questions of multiple Haar and trigonometric series", Math. Notes, 46:2 (1989), 646653.

[6] М. Г. Плотников, "Вопросы единственности для кратных рядов Хаара", Maтем. сб., 196:2 (2005), 97-116; англ. пер.: M. G. Plotnikov, "Uniqueness for multiple Haar series", Sb. Math., 196:2 (2005), 243-261.

[7] П. Л. Ульянов, "О рядах по системе Хаара", Матем. сб., 63(105):3 (1964), 356391.

[8] Б. С. Кашин, А. А. Саакян, Ортогональные ряды, Изд. 2, Изд-во АФЦ, М., 1999; англ. пер. 1-го изд.: В. S. Kashin, А. А. Saakyan, Orthogonal series, Transl. Math. Monogr., 75, Amer. Math. Soc., Providence, RI, 1989.

[9] K. M. Ostaszewski, "Henstock integration in the plane", Mem. Amer. Math. Soc., 63:353 (1986).

[10] Л.В.Линьков, “Эквивалентность различных определений двоичного регулярного интеграла на плоскости", Вестн. МГУ. Сер. 1. Матем., мех., 2000, № 5, 50-53; англ. пер.: L. V. Lin'kov, "Equivalence of two definitions of a double regular integral on the plane", Moscow Univ. Math. Bull., 55:5 (2000), 28-31.

[11] L. Di Piazza, "Variational measures in the theory of the integration in $\mathbb{R}^{m}$ ", Czechoslovak Math. J., 51:1 (2001), 95-110.

[12] B. Bongiorno, L. Di Piazza, V. Skvortsov, "A new full descriptive characterization of Denjoy-Perron integral", Real Anal. Exchange, 21:2 (1995), 656-663.

[13] Z. Buczolich, W.F. Pfeffer, "Variations of additive functions", Czechoslovak Math. J., 47:3 (1997), 525-555.

[14] W.F. Pfeffer, "A descriptive definition of a variational integral and applications", Indiana Univ. Math. J., 40:1 (1991), 259-270. 
[15] W. F. Pfeffer, "Comparing variations of charges", Indiana Univ. Math. J., 45:3 (1996), 643-654.

[16] C.-A. Faure, J. Mawhin, "The Hake's property for some integrals over multidimensional intervals", Real Anal. Exchange, 20:2 (1995), 622-630.

[17] В. А. Скворцов, "Некоторое обобщение интеграла Перрона", Вестн. МГУ. Сер. 1. Матем., мех., 24:4 (1969), 48-51.

[18] М.Г. Плотников, "О восстановлении коэффициентов двумерных рядов Хаара", Изв. вузов. Сер. матем., 2005, № 2, 45-53; англ. пер.: M. G. Plotnikov, "Reconstruction of coefficients of the bivariate Haar series", Russian Math. (Iz. VUZ), 49:2 (2005), 42-50.

[19] М.Г. Плотников, "О единственности всюду сходящихся кратных рядов Хаара", Вестн. МГУ. Сер. 1. Матем., мех., 2001, № 1, 23-28; англ. пер.: M. G. Plotnikov, "The uniqueness of everywhere convergent multiple Haar series", Moscow Univ. Math. Bull., 56:1 (2001), 24-29.

[20] И.П. Натансон, Теория функций вещественной переменной, Лань, СПб., 1999; англ. пер. 1-го изд.: I. P. Natanson, Theory of functions of a real variable, Frederick Ungar Publ. Co., New York, 1955.

\section{М. Г. Плотников (M. G. Plotnikov)}

Вологодская государственная молочнохозяйственная академия

E-mail: mgplotnikov@mail.ru
Поступила в редакцию 26.01.2006 и 03.04.2007 